\title{
Quantitative Measurements of X-Ray Intensity
}

\author{
Michael J. Haugh ${ }^{1}$ and Marilyn Schneider ${ }^{2}$ \\ ${ }^{1}$ National Security Technologies, LLC, \\ ${ }^{2}$ Lawrence Livermore National Laboratory \\ USA
}

\section{Introduction}

This chapter describes the characterization of several X-ray sources and their use in calibrating different types of X-ray cameras at National Security Technologies, LLC (NSTec). The cameras are employed in experimental plasma studies at Lawrence Livermore National Laboratory (LLNL), including the National Ignition Facility (NIF). The sources provide X-rays in the energy range from several hundred $\mathrm{eV}$ to $110 \mathrm{keV}$. The key to this effort is measuring the $\mathrm{X}$-ray beam intensity accurately and traceable to international standards. This is accomplished using photodiodes of several types that are calibrated using radioactive sources and a synchrotron source using methods and materials that are traceable to the U.S. National Institute of Standards and Technology (NIST). The accreditation procedures are described.

The chapter begins with an introduction to the fundamental concepts of X-ray physics. The types of X-ray sources that are used for device calibration are described. The next section describes the photodiode types that are used for measuring X-ray intensity: power measuring photodiodes, energy dispersive photodiodes, and cameras comprising photodiodes as pixel elements. Following their description, the methods used to calibrate the primary detectors, the power measuring photodiodes and the energy dispersive photodiodes, as well as the method used to get traceability to international standards are described. The X-ray source beams can then be measured using the primary detectors. The final section then describes the use of the calibrated X-ray beams to calibrate X-ray cameras. Many of the references are web sites that provide databases, explanations of the data and how it was generated, and data calculations for specific cases. Several general reference books related to the major topics are included. Papers expanding some subjects are cited.

\section{Brief introduction to X-rays: Characteristic spectral lines and bremsstrahlung}

\subsection{Characteristic X-ray spectral lines from atoms}

The electronic structure of an atom, using Ag as an example and shown in Fig. 1, is:

$$
{ }^{47} \mathrm{Ag}: 1 \mathrm{~s}^{2} 2 \mathrm{~s}^{2} 2 \mathrm{p}^{6} 3 \mathrm{~s}^{2} 3 \mathrm{p}^{6} 3 \mathrm{~d}^{10} 4 \mathrm{~s}^{2} 4 \mathrm{p}^{6} 4 \mathrm{~d}^{10} 5 \mathrm{~s}^{1}
$$

Shown in Fig. 1 is the energy level diagram for the lowest four quantum numbers of the $\mathrm{Ag}$ ion, i.e., one of the 1 s electrons, has been removed. How the electron is removed is covered in the next section. 


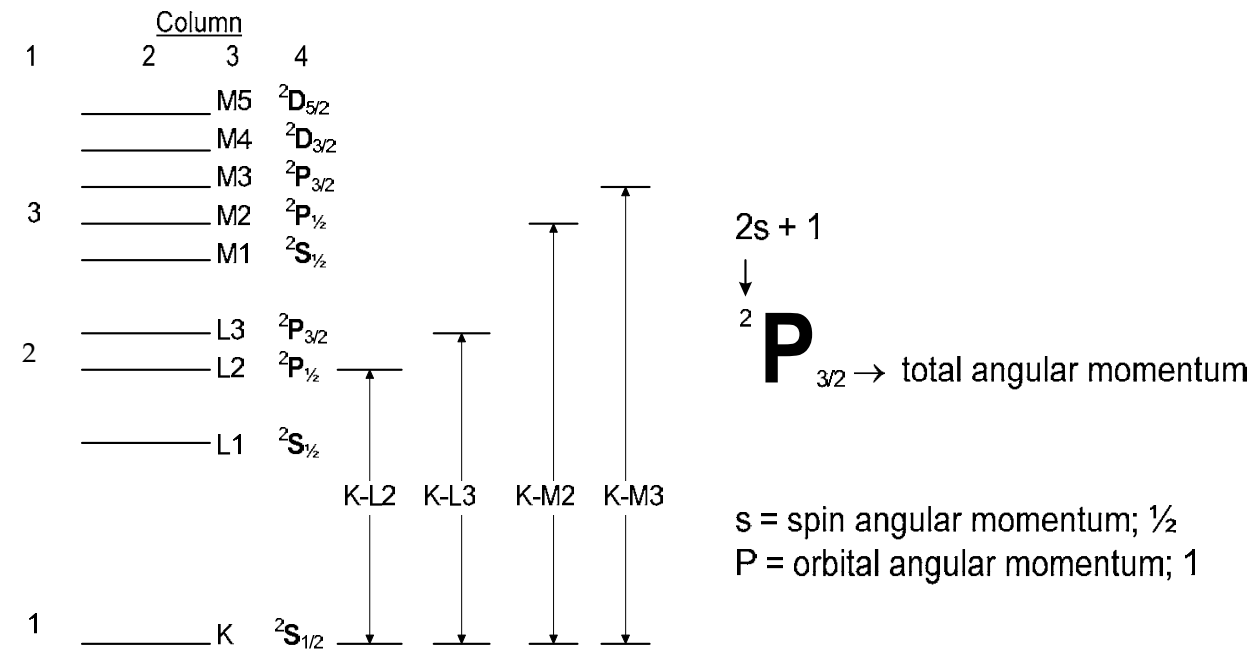

Fig. 1. The energy level diagram for the lowest levels of the singly ionized Ag atom.

Column 1 is the principle quantum number, column 2 indicates an energy level, column 3 is the IUPAC designation for the state, and column 4 is the state electronic structure.

We use the Russell-Saunders angular momentum coupling scheme (Herzberg, 1945) to describe the electronic structure of each state. The notation is illustrated in Fig. 1. The superscript "s" denotes $2 s+1$ where "s" is the total spin of the state. In this case, it is a doublet state since there is an unpaired electron (one electron has been removed). The upper case letter indicates the orbital angular momentum ( $\mathrm{S}$ means zero angular momentum; $\mathrm{P}$ means one unit angular momentum; D means two units, etc.) The subscript indicates the combination of orbital angular momentum and spin angular momentum. This description is somewhat simplified but it gives insight into transition probabilities and what X-ray transitions are expected along with their relative intensities.

When an electron has been removed from the lowest energy level, the ${ }^{2} \mathrm{~S}_{1 / 2}$ state, an electron can drop from a higher level with the simultaneous emission of a photon. The relative energies of these states can be obtained from the binding energies of the electrons in each state, and these are given in Table 1. A good source for this information is the Center for X-Ray Optics web site of the Lawrence Berkeley National Laboratory (CXRO reference).

The readily observed X-ray spectral lines to the electron deficient $\mathrm{K}$ level are shown in Fig. 1 column 4 and the energies and relative probabilities are given in Table 2.

Both the Siegbahn and the newer International Union of Pure and Applied Chemistry (IUPAC) notations for the transitions are given. The IUPAC notation is a bit less obscure but the Siegbahn notation is still more popular in current literature. In the IUPAC notation, the number refers to the order of the energies in the shell as shown in Fig. 1, so that L1 refers to the $n=2 s_{1 / 2}$, L2 refers to the $p_{1 / 2}$, and so on. Note that the spectral emission energy can be estimated by taking the difference between the corresponding binding energy given in Table 1. This estimate is reasonably accurate for $\mathrm{K}$ transitions, but care should be taken when using this estimate for higher energy level transitions. If the electron is removed from the $\mathrm{n}=2$ shell, the spectral emission is referred to as an L line. The set of easily observed emission lines is given in Table 3. 


\begin{tabular}{|c|c|c|}
\hline Energy level & IUPAC & Energy, eV \\
\hline $\mathrm{K} 1 \mathrm{~s}$ & $\mathrm{~K} 1$ & 25514 \\
\hline $\mathrm{L} 2 \mathrm{~s}$ & $\mathrm{~L} 1$ & 3806 \\
\hline $\mathrm{L} 2 \mathrm{p}_{1 / 2}$ & $\mathrm{~L} 2$ & 3524 \\
\hline $\mathrm{L} 2 \mathrm{p}_{3 / 2}$ & $\mathrm{~L} 3$ & 3351 \\
\hline $\mathrm{M} 3 \mathrm{~s}$ & $\mathrm{M} 1$ & 719.0 \\
\hline $\mathrm{M} 3 \mathrm{p}_{1 / 2}$ & $\mathrm{M} 2$ & 603.8 \\
\hline $\mathrm{M} 3 \mathrm{p}_{3 / 2}$ & $\mathrm{M} 3$ & 573.0 \\
\hline $\mathrm{M} 3 \mathrm{~d}_{3 / 2}$ & $\mathrm{M} 4$ & 374.0 \\
\hline $\mathrm{M} 3 \mathrm{~d}_{5 / 2}$ & $\mathrm{M} 5$ & 368.3 \\
\hline $\mathrm{N} 4 \mathrm{~s}$ & $\mathrm{~N} 1$ & 97.0 \\
\hline $\mathrm{N} 4 \mathrm{P}_{1 / 2}$ & $\mathrm{~N} 2$ & 63.7 \\
\hline $\mathrm{N} 4 \mathrm{p}_{3 / 2}$ & $\mathrm{~N} 3$ & 58.3 \\
\hline
\end{tabular}

Table 1. Electron Binding Energies for the Ag Atom

\begin{tabular}{|c|c|c|c|}
\hline $\begin{array}{c}\text { Siegbahn } \\
\text { Designation }\end{array}$ & $\begin{array}{c}\text { IUPAC } \\
\text { Designation }\end{array}$ & $\begin{array}{c}\text { Spectral Line } \\
\text { Energy, eV }\end{array}$ & $\begin{array}{c}\text { Relative } \\
\text { Intensity }\end{array}$ \\
\hline K $\alpha 2$ & K-L2 & 21990.3 & 53 \\
\hline K $\alpha 1$ & K-L3 & 22162.92 & 100 \\
\hline$K \beta 3$ & K-M2 & 24911.5 & 9 \\
\hline$K \beta 1$ & K-M3 & 24942.4 & 16 \\
\hline$K \beta 2$ & K-N2,3 & 25456.4 & 4 \\
\hline
\end{tabular}

Table 2. The K Type X-ray Spectral Lines for the Ag Ion

\begin{tabular}{|c|c|c|c|}
\hline $\begin{array}{c}\text { Siegbahn } \\
\text { Designation }\end{array}$ & IUPAC Designation & $\begin{array}{c}\text { Spectral Line } \\
\text { Energy, eV }\end{array}$ & $\begin{array}{c}\text { Relative } \\
\text { Intensity }\end{array}$ \\
\hline L $\alpha 2$ & L3-M4 & 2978.21 & 11 \\
\hline L $\alpha 1$ & L3-M5 & 2984.31 & 100 \\
\hline L $\beta 1$ & L2-M4 & 3150.94 & 56 \\
\hline L $\beta 2,15$ & L3-N5,6 & 3347.81 & 13 \\
\hline L $\gamma 1$ & L2-N5 & 3519.6 & 6 \\
\hline
\end{tabular}

Table 3. The L Type X-ray Spectral Lines for the Ag Ion

\subsection{Spectral line widths, lifetimes, and competing processes}

The X-ray spectral lines have a narrow width relative to the photon energy. The line widths for several fluorescence transitions in the Ag singly ionized atom are given in Table 4. One can estimate the fluorescence lifetime for the line width using the uncertainty relation given in Equation (1):

$$
\Delta E^{*} \Delta t \geq h
$$

$\Delta \mathbf{E}=\quad$ fluorescence line width, $\mathrm{eV}$

$\Delta \mathbf{t}=\quad$ lifetime of the fluorescence state, sec

$\mathbf{h}=\quad 4.135 \times 10^{-15} \mathrm{eV} \bullet$ s, Planck's constant 
The calculated lifetimes for the Ag transitions are given in Table 4. The fluorescence process can also be treated as a rate of decay from the higher energy state to the lower energy state with the rate constant given as the reciprocal of the lifetime. The rate constants for the two excited states $\mathrm{Ag}^{+}$ion decay is given in Table 4 .

\begin{tabular}{|c|c|c|c|}
\hline Ag Transition & $\begin{array}{c}\text { Line Width, } \\
\mathbf{e V}\end{array}$ & $\begin{array}{c}\text { Lifetime, } \\
\text { sec }\end{array}$ & $\begin{array}{c}\text { Decay Rate Constant, } \\
\text { sec }^{-1}\end{array}$ \\
\hline K-L2 & 8.9 & $4.65 \times 10^{-16}$ & $2.15 \times 10^{15}$ \\
\hline K-L3 & 8.6 & $4.81 \times 10^{-16}$ & $2.08 \times 10^{15}$ \\
\hline L3-M4 & 2.2 & $1.88 \times 10^{-15}$ & $5.32 \times 10^{14}$ \\
\hline L3-M5 & 2.34 & $1.76 \times 10^{-15}$ & $5.66 \times 10^{14}$ \\
\hline L2-M4 & 2.4 & $1.72 \times 10^{-15}$ & $5.80 \times 10^{14}$ \\
\hline
\end{tabular}

Table 4. Line widths and fluorescence lifetimes for several Ag transitions

There are other processes that compete with the fluorescence process. The process that most affects the X-ray fluorescence is called the Auger effect after Pierre Auger, although it was first discovered and published a year earlier by Lise Meitner. The Auger effect describes the transfer of energy that can occur when a vacant state is filled by an electron from the next higher state, but the energy for this transition is transferred to an electron in a higher state which is ejected from the ion, carrying the excess energy as kinetic energy. For example, consider an ion with a hole in the $\mathrm{K}$ shell that is filled by an electron from the L1 state. For the Auger process, the energy from the K-L1 transition is transferred to the L2 electron which is ejected from the ion with kinetic energy equal to the difference between the energy for the K-L1 transition and the binding energy of the L2 electron. The rate constant for this Auger process is about $1 \times 10^{15} \mathrm{sec}^{-1}$. Comparing this rate to the fluorescence rate for the Ag K transitions, we note that the Auger rate is smaller so that the fluorescence yield for that condition will be about $85 \%$ of the of the total rate for filling the hole in the $\mathrm{K}$ shell. For $\mathrm{K}$ transition energies near $9 \mathrm{keV}$ (atomic number near 30 ), the fluorescence decay rate and the Auger rate are about equal and the yields will be about $50 \%$. For lower atomic numbers, the fluorescence yield will be lower than $50 \%$ and conversely, the yield will be larger than $50 \%$ for atomic numbers larger than 30 . Recall that the line width of the transition is determined by the total rate of the excited state decay. For the $\mathrm{Ag}^{+} \mathrm{K}$ transition the fluorescence rate is dominant. For $\mathrm{L}$ transitions, the Auger rate dominates up to the atomic number 100. It is only at this value of $Z$ that the fluorescence yield is $50 \%$. So the line widths for $\mathrm{L}$ transitions are determined by the Auger process and never drop below 2 or $3 \mathrm{eV}$. Refer to graphs of the relative yield as a function of atomic number (Podgorsak, 2010). There are several other internal conversion processes that compete with fluorescence but their rates are much lower and they will not be discussed here. The Auger effect is used for chemical analysis by measuring the kinetic energy of the Auger electron, a technique called Auger electron spectroscopy. The other competing processes also have a niche in analytical chemistry.

\subsection{Electron impact to produce X-rays}

The X-rays used in our measurements are primarily produced by the impact of electrons on solid materials. When an electron moving at a high velocity enters a solid material it 
deposits its energy in the solid in a variety of ways. Most of the energy ends up heating the anode but our interest is in that small percent of interactions that produce X-rays. Our strongest interest is in the collisions that remove an inner electron from the target material and produce the characteristic X-ray spectral lines from the atom. In fact, the X-radiation produced by the interactions of the electron with the solid material is a small fraction of the electron's energy loss processes. As can be seen in the NIST ESTAR tables (ESTAR), the radiation yield from $\mathrm{Ti}$ for electron impact in the energy range of $110 \mathrm{keV}$ is less than $0.5 \%$, and the majority of that radiation is bremmstrahlung. Bremmstrahlung is the spectrum of X-rays produced by the deceleration of electrons. Fig. 2 shows a typical emission produced by electron impact for a $\mathrm{Ti}$ anode target. The transition energies are given in Table 5 .

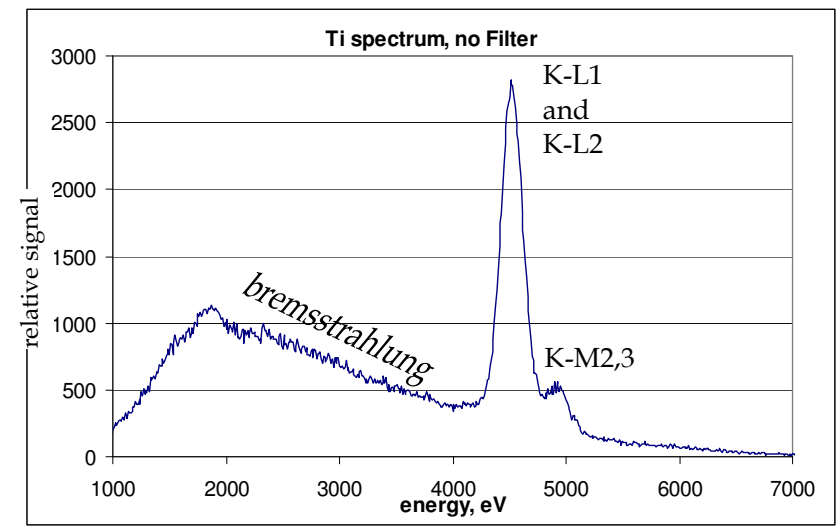

Fig. 2. This is a spectrum of the X-rays produced when an accelerated beam of electrons strikes a Ti anode.

\begin{tabular}{|c|c|c|c|c|}
\hline Transition & K-L2 & K-L3 & K-M2,3 & K-M4,5 \\
\hline Energy, eV & 4504.92 & 4510.899 & 4931.83 & 4962.27 \\
\hline
\end{tabular}

Transition energies are taken from the NIST X-ray database.

Table 5. Ti K X-ray Emission Lines

The anode was at $8,000 \mathrm{~V}$ and the heated filament electron source was near ground potential. The energy dispersive detector that was used to take this spectrum has a resolution near $240 \mathrm{eV}$. The tall, narrow band near $4500 \mathrm{eV}$ comprises the K-L3 and the K-L2 $\mathrm{Ti}$ spectral lines. The spectral lines of $\mathrm{Ti}$ are approximately $2 \mathrm{eV}$ wide. The bremsstrahlung is the broad band ranging from less than $1000 \mathrm{eV}$ to $7000 \mathrm{eV}$ and peaking near $2000 \mathrm{eV}$. The count of photons in the bremsstrahlung is 1000 times larger than the counts in the spectral lines.

The characteristic radiation depends on the anode material properties and the energy of the impacting electron. We have observed that the intensity of emission for characteristic lines follows this equation: 


$$
I_{c}=k\left(V_{e}-V_{b}\right)^{n}
$$

$\mathbf{I}_{\mathbf{c}}=\quad$ intensity of the characteristic $\mathrm{X}$-ray line

$\mathbf{k}=\quad$ proportionality constant

$\mathbf{V}_{\mathrm{e}}=\quad$ accelerating voltage of the electron

$\mathbf{V}_{\mathbf{b}}=\quad$ binding energy of the bound electron

$\mathbf{n}=\quad$ a number somewhat greater than 2

Using this introduction to the basics of X-ray emission, the sources used to produce X-ray emission are presented in some detail in the following section.

\section{X-ray sources}

\subsection{The diode source}

NSTec laboratories have four X-ray sources that cover the X-ray spectral energy range from $50 \mathrm{eV}$ to $110 \mathrm{keV}$. All the primary X-ray sources are the diode type; electrons are emitted from a heated tungsten filament, and then accelerated by an electric field to strike an anode. Two sources use a secondary beam that is generated when the primary beam strikes a sheet of material that fluoresces.

The diode sources produce spectral lines that are characteristic of the anode material and a broad spectrum of radiation known as bremsstrahlung, peaking near one-third of the accelerating voltage. A typical diode source is shown in Fig. 3. The filament is heated by an independent electrical circuit that is near ground potential. The anode is maintained at a high positive voltage so that the electrons emitted from the filament are accelerated and strike the anode at the energy determined by the voltage difference. The electric field is shaped using guide wires. X-rays are emitted in all directions and some exit the aperture, as shown in Fig. 3, and enter into the sample chamber. The anode is water-cooled so that a high beam current can be tolerated, thus giving a strong X-ray intensity. This intensity allows collimation of the X-ray beam with a pair of slits, as well as isolation of individual spectral lines using a diffraction crystal. The narrow band X-ray source can measure sample properties such as filter transmission, crystal reflectivity, and sensor efficiency. The source and sample chamber are in vacuum. The voltage supply is $20 \mathrm{kV}$, making the highest available spectral line nearly $17 \mathrm{keV}$ ( $\mathrm{Zr} \mathrm{K}$ spectral lines).

The other diode source uses anodes that are cooled only by thermal conduction through the mechanical connections. This limits its operation to $10 \mathrm{~W}$ and $10 \mathrm{kV}$, with a usable spectral range from $700 \mathrm{eV}$ to $8400 \mathrm{eV}$. It is often used to measure the absolute efficiency of X-ray cameras and the sensitivity variation across the sensor pixels.

The third source covers the spectral energy range from 8 to $111 \mathrm{keV}$. It uses X-rays from a diode source to produce fluorescent $\mathrm{X}$-rays from a fluorescer material. This source is progressing toward NVLAP accreditation and will be described in detail in the next section. The fourth source is currently being built and will cover the X-ray spectral region from 50 $\mathrm{eV}$ to several $\mathrm{keV}$, also operating on the fluorescer principle.

The NSTec X-ray sources are used to calibrate and characterize components or complete systems that are used in the study of plasmas and similar efforts. A large component of our present calibration efforts is for diagnostics that are used on the NIF target diagnostics.

\subsection{Reducing the band width of the source: filters, grazing incidence mirrors, and diffraction crystals}

The emission from a diode source produced by the impact of the electrons on the anode has a broad band of bremsstrahlung and the characteristic spectral lines from the anode 
composition as was shown in Fig. 2. The large amount of bremsstrahlung X-rays does not allow one to use the raw emission from the diode source to accomplish calibrations such as measuring the energy dependence of a detector's sensitivity. There are several methods for reducing the spectral band width of the raw diode emission: (1) using thin sheets of solid materials that can act as high pass filters; (2) using a high pass filter combined with a grazing incidence mirror to make a band pass filter; (3) using fluorescers that produce only the spectral lines of the fluorescer sheet; and (4) using diffraction crystals to reflect only the $X$-rays that meet the Bragg angle requirements.

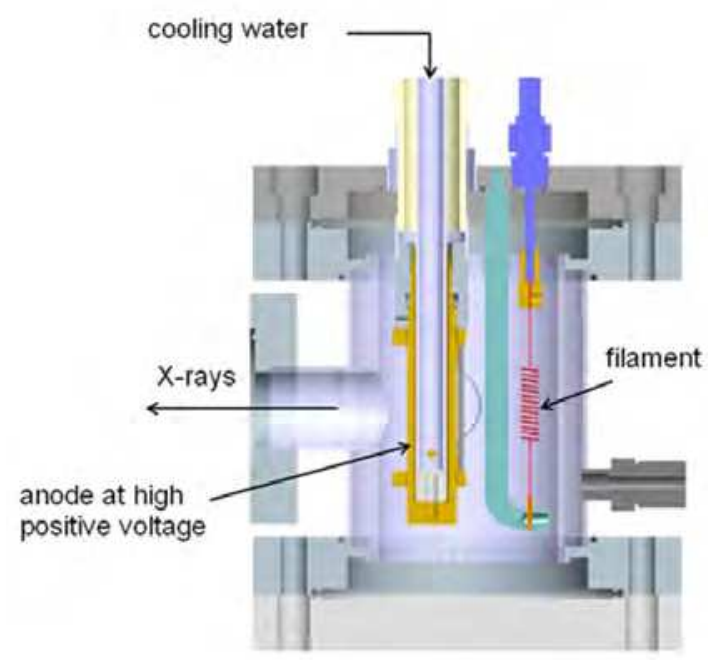

Fig. 3. Example of an X-ray diode

\subsubsection{Filters}

Thin sheets of solid material absorb X-rays and the transmission of the sheets depends upon the $\mathrm{X}$-ray energy, the material thickness, and the atomic number $\mathrm{Z}$ of the material. Gases can also absorb X-rays but are not practical as filters for the applications described in this chapter. The transmission of a Ti sheet that is $25 \mu \mathrm{m}$ thick is shown as Fig. 4(a).

The X-rays are absorbed in the Ti until the X-ray energy gets above $3000 \mathrm{eV}$. At the binding energy of the $\mathrm{Ti} 1$ s electron, $4966 \mathrm{eV}$, referred to as the $\mathrm{K}$ edge, the $\mathrm{X}$-rays are again strongly absorbed. The sheet begins transmitting $X$-rays again when the $X$-ray energy rises above $6000 \mathrm{eV}$. This is the typical behavior of the X-ray transmission for solid materials. The transmission of materials for X-rays up to $30 \mathrm{keV}$ is readily obtained using the CXRO web site. For higher energies, one can obtain absorption cross sections in the NIST tables. The transmission characteristics shown in Fig. 4(a) can be used to make a band pass filter for transmission of the Ti $\mathrm{K}$ lines when the electron accelerating voltage is at $8000 \mathrm{eV}$ or lower and the Ti filter is sufficiently thick. This application will be discussed in more detail in the description of camera calibrations. High pass filters can be made from low Z materials and plastics are the most convenient. The transmission of $400 \mu \mathrm{m}$ thick polyimide is shown in Fig. 4(b). The DuPont version of this material is called Kapton and the material is reasonably resistant to X-ray damage. The $\mathrm{X}$-ray energy at $50 \%$ transmission is near $6 \mathrm{keV}$ and the range 
of X-ray energy for the transmission range from $10 \%$ to $90 \%$ is $6 \mathrm{keV}$. This is a very broad cut off for the high pass filter.

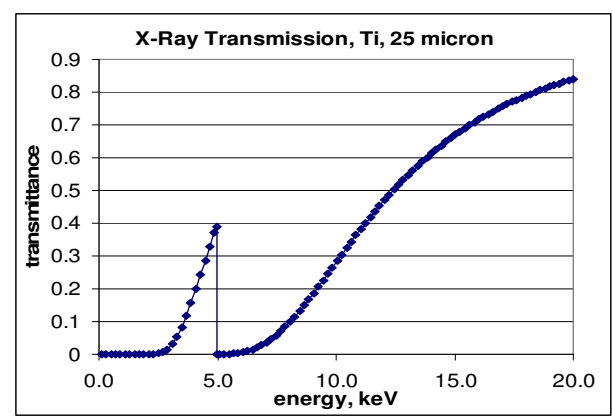

(a)

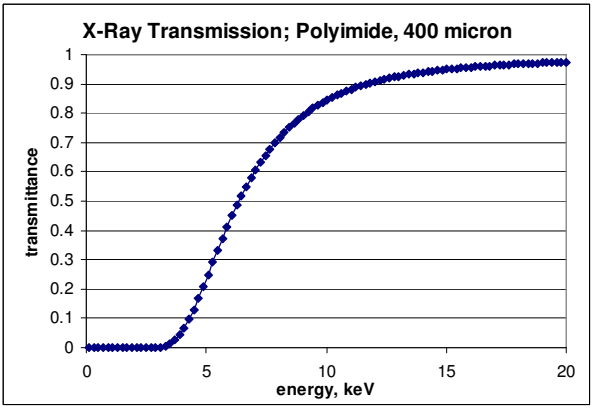

(b)

Fig. 4. Graphs showing the X-ray transmission of (a) a $25 \mu \mathrm{m}$ thick sheet of Ti, $\mathrm{Z}=22$, and (b) a $400 \mu \mathrm{m}$ thick sheet of polyimide.

\subsubsection{Grazing incidence mirror}

In materials, the index of refraction for X-rays is complex, with a magnitude slightly less than 1 . The consequence of this is that an X-ray beam incident from vacuum onto a material is mainly absorbed, unless it is incident at a shallow (grazing) angle to the surface. Since the vacuum is the more optically dense region, the X-ray experiences "total internal reflection" and is specularly reflected. This forms the basis of grazing incidence X-ray mirrors. These mirrors reflect $X$-rays at the specular angle for angles less than a few degrees. As the mirror is rotated with respect to the direction of the X-ray beam, at some angle the reflected intensity will start to decrease and will eventually go to zero reflected intensity. The angle at which the X-ray intensity drops to $50 \%$ of the reflection at very low energies is referred to as the maximum reflection angle. The maximum reflection angle is a function of the X-ray energy, the mirror composition, and the mirror roughness. Calculated reflectivity curves for

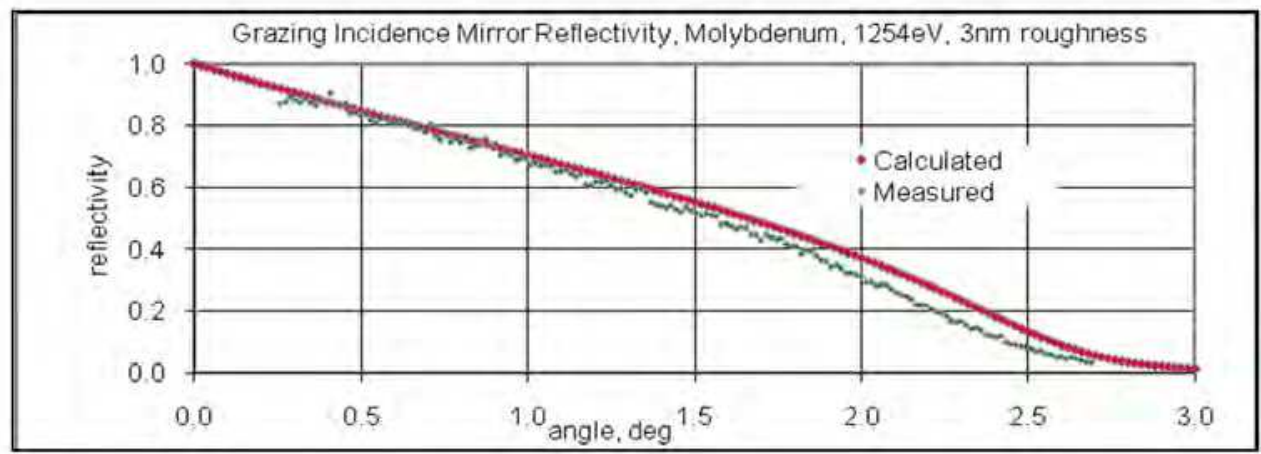

Fig. 5. This graph is a comparison of the measured reflectivity curve for the molybdenum grazing incidence mirror at an X-ray energy of $1254 \mathrm{eV}$ with the calculated reflectivity with a surface roughness of $3 \mathrm{~nm}$ rms. 
various materials and surface roughness can be obtained from the CXRO web site. A typical measured grazing incidence reflectivity curve is shown in Fig. 5 (green scatter). The corresponding calculated reflectivity curve is shown in red in Fig. 5. Given their angular dependence, grazing incidence mirrors are often used as low pass filters. The combination of a grazing incidence mirror with an appropriate thin sheet filter described previously forms a band pass filter.

The reflectivity curve for a grazing incidence mirror is affected by materials adsorbed on the surface. Water vapor and oxygen can significantly affect the reflectivity curve. For this reason, the grazing incidence mirror reflectivity curve is usually calibrated before it is used in experimental applications. This can be done using the NSTec sources. The synchrotron at Brookhaven is also used for these calibrations.

\subsubsection{Diffraction crystal}

Crystals are often used to isolate individual spectral lines from a diode source. They are used in plasma diagnostics as components of a spectrograph. The crystal reflectivity follows the Bragg law for the location of the maximum reflection as a function of X-ray energy:

$$
\mathrm{n}(12398.425 / \mathrm{E})=2 \mathrm{~d} \sin \Theta
$$

$\mathbf{n}=\quad$ an integer equal to the diffraction order

$\mathbf{E}=\quad$ X-ray energy, $\mathrm{eV}$

$\mathbf{d}=\quad$ distance between the crystal planes, $\AA$

$\Theta=\quad$ angle between the $X$-ray beam and the crystal plane

For $\mathrm{n}=1$ and a given $\Theta$, only the X-rays having the energy E given by the Bragg law will be reflected. For a monochromatic plane wave the Bragg reflection curve has a finite width. Theoretical calculations of the reflection curves for many crystals can be obtained at the Argonne web site (Stepanov, 1997 \& 2009). Real crystals can approach this theoretical width if properly made. Two of the NSTec sources have the ability to measure the reflectivity curve of flat and curved crystals such as those made of mica. (Haugh \& Stewart, 2010) The use and calibration of crystals is not covered in this chapter.

\subsection{The Manson type diode source: an X-ray system used for calibration}

One of the NSTec diode type X-ray sources that is used for testing and calibrations generates $\mathrm{X}$-rays in the energy range from $400 \mathrm{eV}$ to $9 \mathrm{keV}$. We refer to this as the Manson source since this was the manufacturer. The source is not water cooled, and the power is limited to $10 \mathrm{~W}$ to avoid melting the anodes. The filament is shaped to a point near the anode. This produces a small spot, approximately $1 \mathrm{~mm}$ diameter, where the electrons impact the anode. This small X-ray emission spot acts as a point source providing a flat X-ray intensity in the sample region allowing us to do radiographic type measurements and to measure the sensitivity variation across the sensor array of a camera.

Fig. 6 shows a schematic diagram of the NSTec Manson system, looking down on it from above. The Manson comprises three compartments: the source chamber and two testing chambers which are the rectangular boxes in the figure. The two test chambers are connected to the main chamber by stainless steel vacuum components that include an isolation gate valve and a mechanical shutter. The diagnostic that is shown attached to the top arm in the figure is at vacuum. Components, such as filters, can be mounted inside the chamber 
Each test chamber has its own vacuum pump and controls and can be isolated from the source chamber by a gate valve, then brought to atmosphere. Test chambers have photodiode and an energy dispersive detectors for measuring X-ray flux and the X-ray spectrum, mounted on push rods so that they can be moved into or out of the beam.

The X-ray beam paths that are used for testing are shown in red in Fig. 6. Filter 1, shown in the source chamber, is used to isolate a narrow wavelength band of $X$-rays. These filters are mounted in a vertical stalk that holds up to three filters. A light blocker prevents visible light emitted by the filament from entering the test chamber which would overwhelm the detectors and CCD.

The Manson system is a multi-anode device, holding up to six different anodes on a hexagonal mounting bracket. Two X-ray beams are isolated from the anode emission for use in the test chambers. A typical X-ray emission produced by the impact of electrons with a metal anode was shown in Fig. 2. The Ti spectrum that is observed when a $100 \mu \mathrm{m}$ thick Ti filter is placed between the X-ray source and the detector as a band pass filter is shown in Fig. 7. See also Fig. 4(a) for the spectral characteristic of a thin sheet of Ti. Comparing the unfiltered Ti spectrum shown in Fig. 2 with the filtered spectrum shown in Fig. 7, we can see that the transmission is now limited to the spectral energy range between $4000 \mathrm{eV}$ and 4966 $\mathrm{eV}$, the latter being the $\mathrm{K}$ edge of Ti. The spectral content now includes the Ti K lines and the bremsstrahlung within the energy range given.

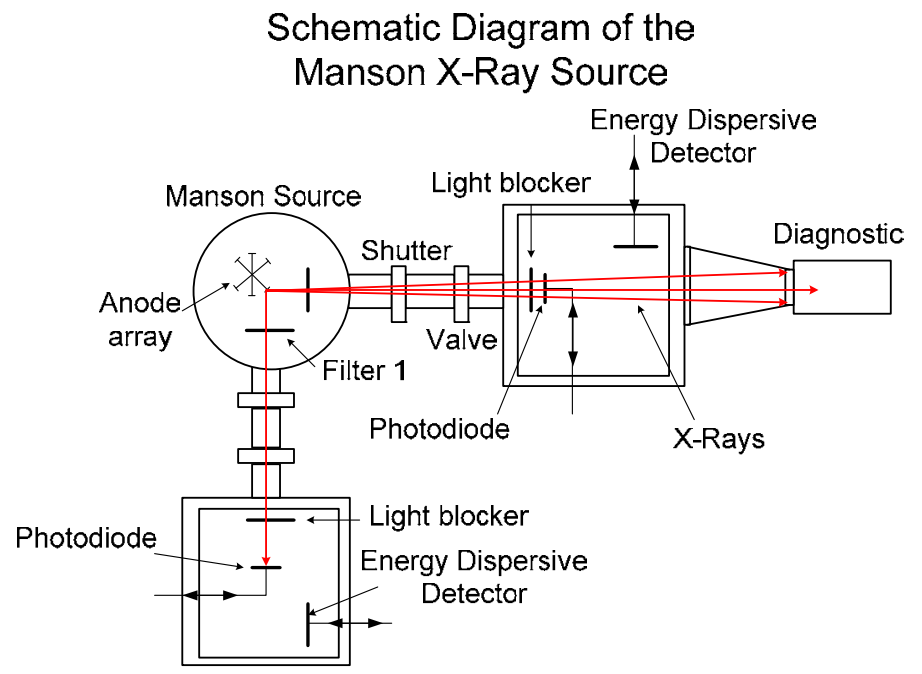

Fig. 6. Manson Schematic. The diagnostic being calibrated is shown directly attached to the chamber at the end of the upper arm. The red lines are the X-ray beam path. 


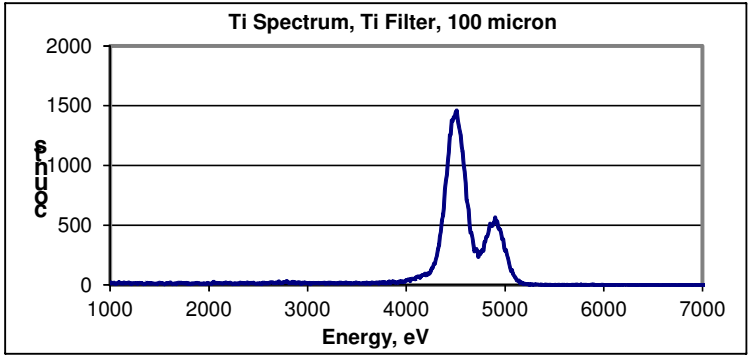

Fig. 7. The spectrum of Ti X-rays shown in Fig. 2 using a Ti filter 100 micron thick to limit the spectral bandwidth

\subsection{Fluorescer source}

The High Energy X-ray system (HEX) uses a diode type source to produce monochromatic $\mathrm{X}$-rays. X-rays from the diode (a commercial $160 \mathrm{kV}$ X-ray tube) excite characteristic X-ray lines in the fluorescer foil. The X-ray tube and the fluorescing targets are enclosed in a lead box. An exit collimator in the lead box shapes the X-rays into a beam. The fluorescer operation is illustrated by Fig. 8. For this example, the fluorescing material is a thin lead $(\mathrm{Pb})$ sheet, with a thickness of approximately $250 \mu \mathrm{m}$, and the filter is a thin platinum (Pt) sheet. Table 6 gives the properties of the fluorescer and filter. The high energy X-ray lines are transmitted by the filter but the low energy lines are stopped by the filter.

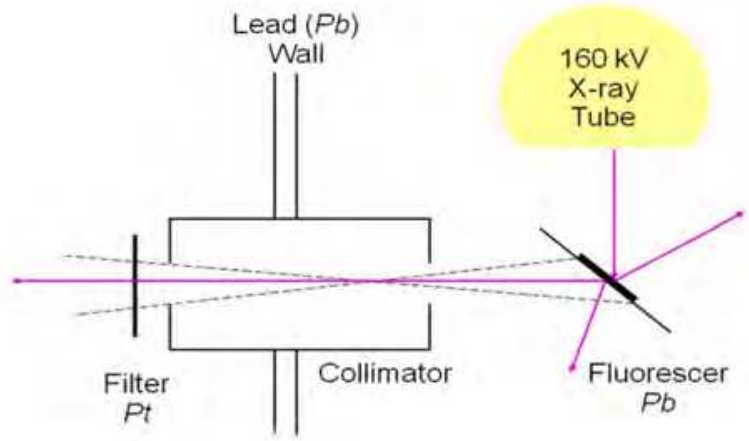

Fig. 8. Illustration of fluorescence principle

\begin{tabular}{|c|c|}
\hline Filter & Fluorescer \\
\hline Platinum $(\mathrm{Pt}), 50 \mu \mathrm{m}$ thick & Lead $(\mathrm{Pb})$ \\
\hline
\end{tabular}

\begin{tabular}{|c|c|}
\hline Transmission & Spectral Lines, $\mathbf{k e V}$ \\
\hline 0.72 & 73,75 \\
\hline 0.40 & 85 \\
\hline $2 \times 10^{-5}$ & $10.4,10.6,12.6,14.8$ \\
\hline 0 & 2.3 \\
\hline
\end{tabular}

Table 6. X-ray Fluorescence 
This method provides a reasonably narrow spectral energy that can be used to calibrate detectors at a range of well defined energies. The resulting spectrum from the arrangement is shown in Fig. 9.

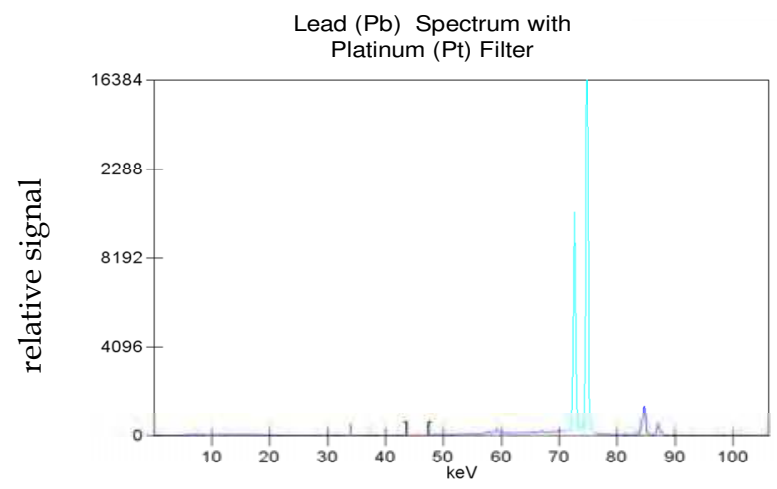

Fig. 9. Pb Spectrum, Pt Filter

The arrangement of the components is shown in Fig. 10(a) and (b).

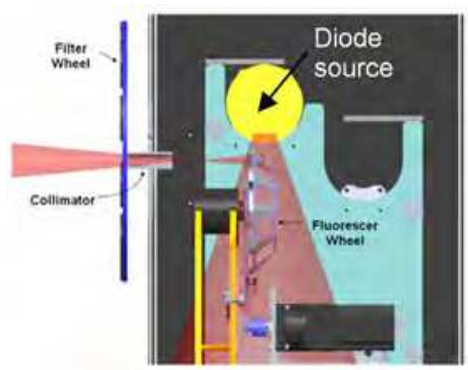

(a)
$\mathrm{Pb}$ chamber containing the diode source and the fluorescer wheel Filter Wheel

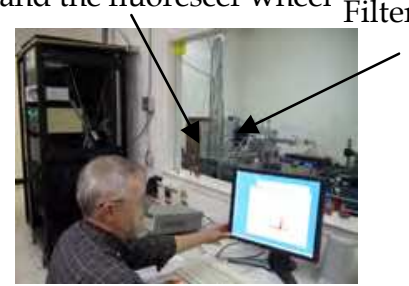

(b)

Fig. 10. (a) HEX source component inside the Pb chamber and (b) a view of the control room looking through a window at the HEX optical table.

The end of the commercial X-ray tube is shown in yellow. The pink trapezoid that starts at the tube represents the primary $X$-ray beam. The fluorescers are mounted on the motorized wheel in the rectangles shown on the wheel. The fluorescer emits in all directions, but the X-ray beam is defined by the collimator inserted into the wall of the lead box, and the beam path is illustrated by the pink triangles. There is a filter wheel mounted downstream from the collimator, and it is also motorized. The fluorescer and the filter can be set from the computer in an adjacent control room, as shown in Fig. 10(b). The fluorescer is usually a thin sheet made of elemental metal, but metal compounds are sometimes used. The maximum intensities obtained when an $11.5 \mathrm{~mm}$ diameter collimator is used are on the order of $1 \times 10^{6}$ photons per $\mathrm{cm}^{2}$ per second, at one meter from the fluorescer, depending on the fluorescer material. The spectral lines used range from $8 \mathrm{keV}$ to $115 \mathrm{keV}$.

Remote adjustment of the fluorescer wheel and the filter wheel is done through the control room computer. Data from the detectors and devices being calibrated are received in the control room. 
The HEX source sits at the end of an optical table as shown in Fig. 11. The sample and the detectors are mounted on the optical table. The control room is separated from the HEX laboratory, as seen in Fig. 10(b).

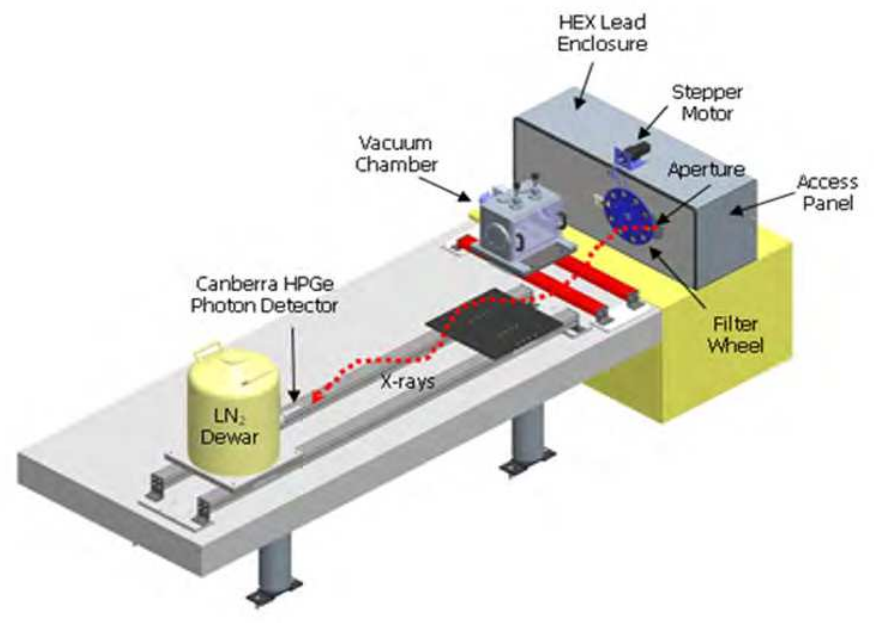

Fig. 11. HEX Layout

\section{Solid state detectors}

\subsection{Introduction to semiconductor detectors}

In the field of X-ray measurements, the term solid state detector usually refers to semiconductor type detectors. There are other X-ray detectors that are solid materials, such as scintillator -photomultiplier combinations, but for this work the term solid state detectors will include only semiconductor detectors. There are 3 types of semiconductor materials used for the work described in this chapter: silicon (Si), germanium (Ge), and cadmium telluride (CdTe). A semiconductor is defined as a material that has a small band gap (which can be manipulated) between the valence electrons and the conduction band, on the order of $1 \mathrm{eV}$ to several $\mathrm{eV}$. A metal has electrons populating the conduction band at any temperature, and an insulator has a large gap, on the order of $10 \mathrm{eV}$ and higher. At normal temperatures, the semiconductor will have some population of electrons in the conduction band, and corresponding holes (positive charge where the electron vacated) in the valence band. The ratio of electrons in the conduction band to those in the valence band is given by the Boltzmann probability relation:

$$
\mathrm{N} / \mathrm{N}_{0}=\mathrm{CT}^{3 / 2} \mathrm{e}^{-(\mathrm{E} / \mathrm{kT})}
$$

$\begin{array}{ll}\mathbf{N}= & \text { population of electrons in the conduction band } \\ \mathbf{N}_{0}= & \text { population of electrons in the valence band } \\ \mathbf{E}= & \text { band gap energy, } \mathrm{eV} \\ \mathbf{k}= & \text { Boltzmann constant, } 8.617343 \times 10^{-2} \mathrm{ev} / \mathrm{K} \\ \mathbf{T}= & \text { Absolute temperature, } \mathrm{K} \\ \mathbf{C}= & \text { A material property }\end{array}$


Consider an X-ray photon incident on the semiconductor. It has an energy that is many times that of the band gap. It interacts with the semiconductor material, primarily through the photoelectric effect, to produce energetic free electrons. These then produce electron-hole pairs. The number of electron-hole pairs produced is proportional to the energy of the X-ray photon. In general it takes several $\mathrm{eV}$ to produce an electron-hole pair, the exact energy depending on the semiconductor material. The number of electron-hole pairs produced by an $\mathrm{X}$-ray photon depends upon the material and the X-ray photon energy as given by:

$$
\mathrm{N}_{\mathrm{e}-\mathrm{h}}=\mathrm{eV} / \varepsilon
$$

$\mathbf{N}_{\mathrm{e}-\mathrm{h}}=\quad$ number of e-h pairs produced

$\mathbf{e V}=\quad$ X-ray photon energy, $\mathrm{eV}$

$\varepsilon=\quad$ energy required to form an e-h pair in the semiconductor material

$\varepsilon$ is usually referred to as the "ionization energy." Table 7 gives the ionization energy and the semiconductor band gap for the three materials we are discussing. The electron-hole pair formed will drift apart. But if a voltage is applied, the electrons will move in the direction opposite to the electric field, and the holes will move in the direction of the electric field, so the charges can be collected and measured.

The room temperature population of electron-hole pairs of the semiconductor (Eq. 4) will be noise in the measurements. Details of semiconductor behavior can be found in Knoll (2001), which describes the physics and behavior of semiconductor detectors.

\begin{tabular}{|c|c|c|}
\hline $\begin{array}{c}\text { Semiconductor } \\
\text { material }\end{array}$ & $\begin{array}{c}\boldsymbol{\varepsilon} \text {, Energy required for one } \\
\text { hole/pair production, eV }\end{array}$ & $\begin{array}{c}\text { Semiconductor band } \\
\text { gap, } \mathbf{e V}\end{array}$ \\
\hline $\mathrm{Si}$ & 3.63 & 1.116 \\
\hline $\mathrm{Ge}$ & 2.96 & 0.665 \\
\hline $\mathrm{CdTe}$ & $4.43^{*}$ & 1.44 \\
\hline
\end{tabular}

Table 7. Properties of semiconductors discussed in this section ( ${ }^{*}$ Quaranta, 1969)

There are several ways that the signal is lost and the solid state detector output fails to give the full measure of the X-ray intensity. Electrons produced by the X-ray interaction can recombine with another hole and electron lost from the charge collection process. Charge can be trapped by impurities or lattice defects and enhance the recombination process. For a properly designed semiconductor detector, this problem can be minimized. A semiconductor typically has a coating on the surface that protects the active semiconductor material from damage by the environment. This surface coating can absorb some of the X-ray beam and this will not generate a current out of the detector. This front surface absorption will have greater impact for the lower energy X-ray photons. For the higher energy photons, at some energy they will begin to pass through the detector's active material and will not be detected. Another signal loss mechanism occurs when the X-ray energy rises above the $\mathrm{K}$ edge of the semiconductor active material. The semiconductor can fluoresce and this energy is lost from the detector.

\subsection{Photodiode used to measure X-ray power}

Calibrated photodiodes are used to measure the $X$-ray power of $X$-ray sources. The sources are used to calibrate $X$-ray detectors. This description of photodiode operation will use the photodiode design that was used to measure the efficiency of X-ray CCD cameras and other 
imaging and non-imaging detectors. The photodiode is made from $\mathrm{Si}, 55 \mu \mathrm{m}$ thick. If all of the X-ray photon energy is deposited in the active $\mathrm{Si}$, and $100 \%$ of the e-h pairs formed are collected, the diode current $\mathbf{i}$ is given in Equation (6) as:

$$
\mathrm{i}=\mathrm{F} \cdot(\mathrm{eV} / \varepsilon) \cdot\left(1.6 \times 10^{-19}\right) \mathrm{amp}
$$

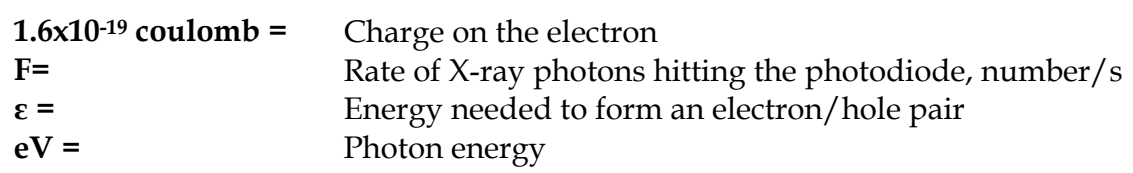

Applying Equation (6) to a Si diode with a $1 \mathrm{~cm}^{2}$ area, with an incident beam of Ti Ka1 $(4510.84 \mathrm{eV})$ X-rays, with a photon intensity of $2 \times 10^{5}$ photons ${ }^{\bullet} \mathrm{cm}^{-2} \bullet \mathrm{s}^{-1}$, yields a current of $39.8 \times 10^{-12} \mathrm{~A}$. This can be readily measured using a commercial picoammeter.

The silicon photodiode in use was designed to measure X-ray intensity with nearly $100 \%$ efficiency for X-ray energies up to about $5 \mathrm{keV}$ (IRD). Several of these photodiodes have been calibrated from $1000 \mathrm{eV}$ to $60 \mathrm{keV}$ at the Physikalisch-Technische Bundensanstalt (PTB Reference, Gottwald, 2006)). Within the measurement uncertainty, which is near $1 \%$, the silicon photodiode is $100 \%$ effective up to the Si K edge, where it dropped several percent. The efficiency rises back to near $100 \%$ by $3 \mathrm{keV}$. The $55 \mu \mathrm{m}$ thick Si photodiode begins to transmit near $5 \mathrm{keV}$ X-ray energy, and at higher energies the diode efficiency follows the Si transmission curve.

\subsection{Energy dispersive photodiodes}

Some photodiodes are designed to measure the energy of the X-ray photons. The types of semiconductor materials that are commonly used include $\mathrm{Si}, \mathrm{Ge}$, and $\mathrm{CdTe}$. A bias voltage is applied to the semiconductor and the electric fields generated require cooling of the detector. The voltage pulses produced by an individual X-rays are amplified and then counted according to pulse height. Pulses that have heights within a certain range are effectively assigned to channels according to the average pulse height by a processor referred to as a multi-channel analyzer. This produces an energy spectrum of the detected Xray photons. The resolving power of the energy dispersive detector is generally limited to several hundred $\mathrm{eV}$.

The detector sensitivity falls off at lower energies due to absorption at the front surface by a "dead" layer of the sensor and/or a window separating the vacuum chamber containing the detector from the environment. It falls off at higher energy when the photons begin to be transmitted by the sensor. The Ge and CdTe detectors are designed to operate optimally in the $10 \mathrm{keV}$ to slightly over $100 \mathrm{keV}$. The $\mathrm{X}$-ray photon interacts with the sensor material in ways other than forming electron-hole pairs, which can reduce its sensitivity. If a photon has sufficient energy, it can knock out a 1s electron of the sensor material, a higher state electron can transition down into the 1s vacancy. A second photon having an energy equal to the energy between these two states can then be emitted. The sensor then produces a corresponding pulse of electron-hole pairs that is smaller than the base peak by the energy difference between the incoming X-ray photon and the binding energy of the sensor material $1 s$ electron. This is referred to as an "escape peak," and effectively this means the incoming X-ray photon is not counted. There are other losses that are introduced by the detector fabrication details.

The Ge detector made by Canberra uses a high purity Ge disk, $8 \mathrm{~mm}$ diameter and $5 \mathrm{~mm}$ thick, that is cooled to liquid nitrogen temperature. The sensor is in a vacuum chamber that 
has a 4 mil thick beryllium (Be) window for X-ray beam entry. Ge has an escape peak near $11.1 \mathrm{keV}$. Fig. 12(a) shows a spectrum from the radioactive isotope of americium (Am) having an atomic mass of 241 (Am-241). This source emits gamma radiation (X-rays that are produced by nuclear transitions) at $59.5 \mathrm{keV}$ and $26.4 \mathrm{keV}$, and X-rays that are produced by electronic transitions from the Am decay daughter neptunium $(\mathrm{Np})$ ion are seen in the $13 \mathrm{keV}$ to $22 \mathrm{keV}$ range.

The CdTe sensor is a $5 \mathrm{~mm}$ square that is $1 \mathrm{~mm}$ thick. It is cooled sufficiently using a thermoelectric cooler so that a $400 \mathrm{~V}$ bias voltage can be applied without electrical breakdown. It also operates in vacuum with a 0.001 inch Be window. The energy required to form an electron-hole pair for this material is $4.43 \mathrm{eV}$. (Herzberg, 1945) Escape peaks occur near $27 \mathrm{keV}$ and $23 \mathrm{keV}$. Electron-hole pairs formed near the back contact of the detector cause fluctuations in pulse height, and they are not seen as belonging to the true peak. This is a loss in sensitivity. The Am-241 spectrum for the CdTe detector is given in Fig. 12(b). Note that there are several peaks in the $30 \mathrm{keV}$ to $40 \mathrm{keV}$ energy range that are not in the spectrum when the Ge sensor is used. A small escape peak is seen near $32 \mathrm{keV}$, and a larger escape peak is seen near $36 \mathrm{keV}$.

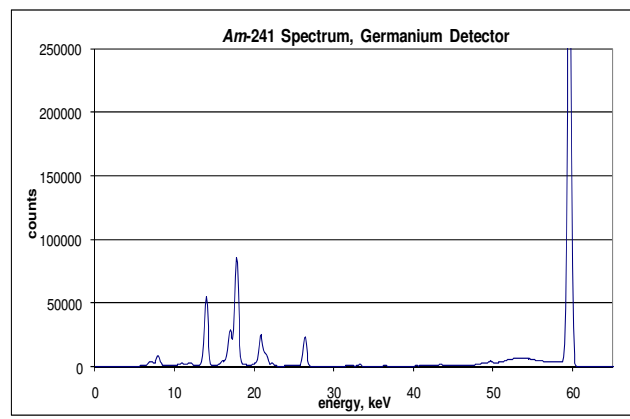

(a)

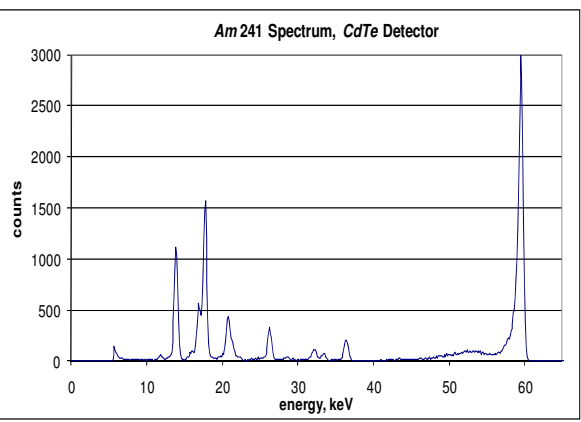

(b)

Fig. 12. Am-241 spectrum. (a) Ge detector, showing $\gamma$-rays (gamma rays) at $59 \mathrm{keV}$ and 26 $\mathrm{keV}$, X-rays from $\mathrm{Np}$ from $22 \mathrm{keV}$ to $14 \mathrm{keV}$, and escape peaks below $14 \mathrm{keV}$. (b) CdTe detector, showing $X$-rays and $\gamma$-rays as in (a), and escape peaks in the $30-40 \mathrm{keV}$ range.

\subsection{Imaging devices}

X-ray imaging is used to obtain geometric and qualitative intensity information in many applications such as tooth damage, stellar luminosity, and temperature and density of laserproduced plasmas. In the past few decades, several types of solid state detectors have replaced film in most imaging applications. These include charge-coupled devices (CCDs), charge-injection devices (CIDs), image plates (photostimulable phosphor plates - PSP), and other devices and sensor materials. After a brief description of image plates, the work described in this chapter will be limited to the CCD and CID Si-based sensors.

\subsubsection{Image plates}

Image plates use principle of photostimulated luminescence (PSL) to to read the X-ray image after the image plate has been exposed. The exposed image is read and digitized with an image plate reader and stored in the computer as an image file. The image plate contains 
very small crystals $(\sim 5 \mu \mathrm{m})$ of barium fluorobromide phosphor with a trace amount of europium as a color center (Maddox, 2011). When exposed to X-rays, an atom of $\mathrm{Eu}^{2+}$ is converted to $\mathrm{Eu}^{3+}$ and the free electron is trapped in the barium fluorobromide lattice, creating a metastable state. The metastable electrons are freed by stimulation from the laser in the image plate reader, and they recombine with the $\mathrm{Eu}^{3+}$ to give off a blue-violet fluorescence that is recorded by the image plate reader with a suitable detector. The digitized image can then be analyzed for geometric information and quantitative intensity information. We now use image plates extensively for characterizing the X-ray beam in a variety of arrangements. We have also calibrated image plates for quantitative intensity measurements that are then applied to plasma studies at LLNL (Maddox, 2011). LLNL and other groups have also evaluated their performance for medical imaging (AAPM, 2006). Image plates will not be considered further in this chapter since the subject of the book is photodiodes.

\subsubsection{Silicon based cameras}

The basic sensing function is the same for all Si-based sensors, and a camera operates in a manner similar to the principles described for the X-ray photon detectors described earlier. The photons interact with the Si semiconductor to produce electron hole pairs. The camera types vary in the method in that the charge is moved from the sensor pixel and eventually produces a digital output proportional to the charge formed. Janesick (2000) is a good source for the details of camera readout methods.

The charge coupled device (CCD) transfers the charge from a row of the sensor pixels to an adjacent row until it reaches the "read-out"row. The charge is read from each pixel sequentially in that "read-out" row as voltage. The voltage is then converted to digital counts. A major advantage of the CCD camera is low electronic noise because it is usually cooled to minimize the dark current from thermally-produced electron-hole pairs (Eq. 4). The major contribution to the noise is then from the read-out. The read-out noise is quite low, typically no more than a few counts. The dominant noise contribution for X-ray applications is then what was produced by the arrival time statistics of the photons themselves, a Poisson distribution. The standard deviation per pixel is then the square root of the number of X-ray photons the pixel absorbed during the exposure time.

An alternative electronic method for reading the charge stored in the individual pixels is the CID. Every pixel can be individually addressed using indexing row and column electrodes. A displacement current proportional to the stored signal charge is read when stored "packets" are shifted between capacitors within individually selected pixels. The displacement current is amplified and converted to a voltage, and fed to the outside world as a digitized signal. The CID technology offers certain advantages over CCD technology such as cost, reduced heat generation, and resistance to blooming. The disadvantage is the high readout noise. Improvements continue in this area but for X-ray photon intensity measurements, the readout noise far exceeds the photon statistical noise for a single image. When calibrating the CID, this can be mitigated by multiple imaging.

Most CCD cameras are front illuminated, the front side being where the gates are located. This region is not active to photon detection. This dead region will stop low energy X-rays and the front illuminated CCD cameras lose sensitivity as the X-ray energy drops below $2000 \mathrm{eV}$. Below $1000 \mathrm{eV}$ they are not useful. For this reason many X-ray CCD cameras are back illuminated and back thinned and are sensitive even into the vacuum ultraviolet. The Si active region is typically $15 \mu \mathrm{m}$ to $30 \mu \mathrm{m}$. The Si thickness thinning does reduce the 
sensitivity at the high energy side. Camera efficiency measurements for both front illuminated and back illuminated cameras are given in Section 6.

\section{Calibrating primary detectors using radioactive sources and a synchrotron source}

\subsection{The calibration concept using radioactive sources}

Radioactive sources provide a variety of spectral lines at well defined energies, as was described in the energy dispersive detector section (section 4.3). The photon output is directly proportional to the activity of the radioactive source, and the activity measurement is traceable to NIST. The uncertainty for the activity is provided by the vendor. The activity $\mathbf{R}$ at time $t$ is given by:

$$
R=R_{0} \exp \left((t \ln 2) / \tau_{1 / 2}\right)
$$

$\mathbf{R}_{\mathbf{0}}$ activity at time $t=0$ (disintegrations $/ \mathrm{sec}$ )

$\tau_{1 / 2} \quad$ radionuclide half life

The radioactive source is placed at some distance from the detector that is sufficiently large so that the source intensity is uniform over the detector area. The power $\Omega$ at the detector in photons per second for a selected spectral band is given by:

$$
\Omega=R B\left(\mathbf{A}_{d} / 4 \pi r^{2}\right) \mathrm{T}
$$

B branching ratio

$\mathbf{A}_{\mathbf{d}}$ detector area

$r$ distance between radioactive source and the detector

T X-ray transmittance through $r \mathrm{~cm}$ of air

The branching ratio is the fraction of nuclear decays that produce the selected spectral band. It has an experimental uncertainty associated with it. If the activity was measured using a gamma emission, and the same gamma is used for the detector calibration, the uncertainty is that given is that given by the vendor in his activity certification. The detector efficiency $\eta$, is then given by:

$$
\eta=S / \Omega
$$

$\mathbf{S}$ is the photon count per second as measured by the detector. The measurement arrangement is shown in Fig. 13. The instrument to be calibrated, the Ge detector, is seen on the right facing the radioactive source. An optical distance meter is located at the far left of the optical rail and is also at the same height as the source and detector and measures the distance from the radioactive source and to the detector window. The internal distances for source and detector are provided by the manufacturers to an accuracy of $0.5 \mathrm{~mm}$ or better. The distance sensor has an accuracy of $\pm 1 \mathrm{~mm}$ and was calibrated at NIST within a month of the detector calibration measurements. Thus the source-to-detector distance accuracy is \pm 1 $\mathrm{mm}$.

\subsection{Measurement results}

Measurement results are given in this section. The spectral lines that were used for these measurements are given in Table 8. 


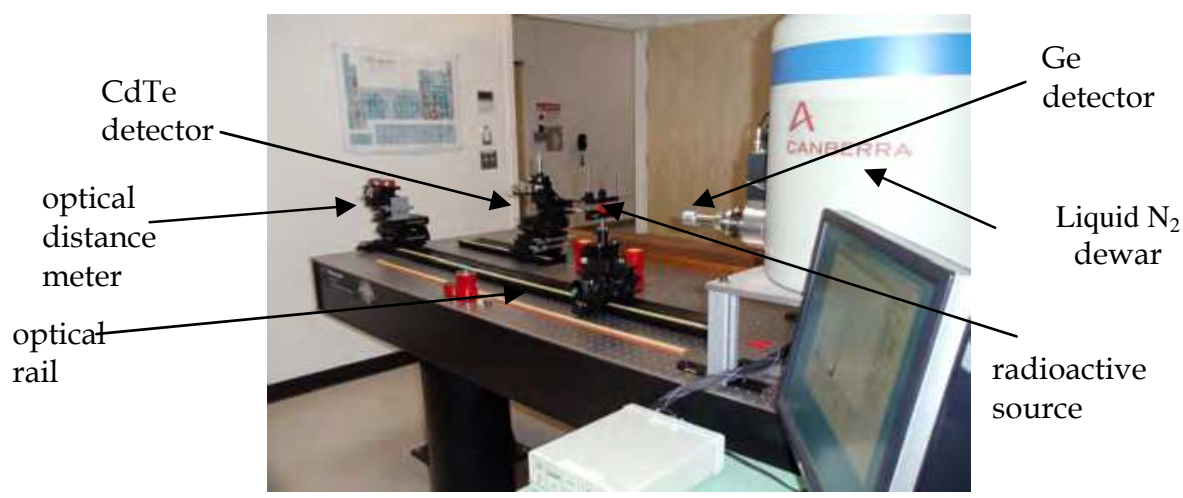

Fig. 13. Experimental arrangement for calibrating detectors using radionuclides

\begin{tabular}{|c|c|c|}
\hline Spectral Energy, keV & Spectral Type & Radionuclide \\
\hline 5.97 & $\mathrm{Mn}, \mathrm{K}$ line & $\mathrm{Fe} 55$ \\
\hline 22.16 & $\mathrm{Ag}, \mathrm{K}$ line & $\mathrm{Cd} 109$ \\
\hline 26.34 & $\gamma$ & $\mathrm{Am} 241$ \\
\hline 41.3 & $\mathrm{Eu}, \mathrm{K}$ line & $\mathrm{Gd} 153$ \\
\hline 59.54 & $\gamma$ & $\mathrm{Am} 241$ \\
\hline 88.00 & $\gamma$ & $\mathrm{Cd} 109$ \\
\hline 97.43 & $\gamma$ & $\mathrm{Gd} 153$ \\
\hline 103.18 & $\gamma$ & $\mathrm{Gd} 153$ \\
\hline
\end{tabular}

Table 8. Spectral Lines Used for the Calibration of the Ge and CdTe Detectors

Quantum efficiency measurements for the Ge detector are shown in Fig. 14(a), and Fig. 14 (b) for the CdTe detector. These measurements show a precision near 3\% at the $95 \%$ confidence level. The Ge detector shows a peak efficiency near $60 \mathrm{keV}$ and falls off in efficiency at lower and higher spectral energies. The CdTe detector has a peak efficiency near $30 \mathrm{keV}$ and also falls off in efficiency at lower and higher spectral energies.

It has been described earlier that escape peaks occur above the K edges (minimum energy needed to remove a $1 \mathrm{~s}$ electron) of the detector materials, and this reduces the detector efficiency. This effect has been directly observed in the calibration of a Si photodiode in the vicinity of the Si K edge (1.39 keV). The K edges for $\mathrm{Ge}, \mathrm{Cd}$, and Te are $11.1 \mathrm{keV}, 26.71 \mathrm{keV}$, and $31.81 \mathrm{keV}$ respectively. (CXRO) In Fig. 14 (a) and (b), note the large gaps that exist between points. The situation regarding the large gaps can be improved by using more radionuclides but there are availability and economic restrictions that will limit this approach. We plan to fill in these energy gaps by calibrating an Si detector up to $60 \mathrm{keV}$ using a synchrotron as described in the next sub-section. This calibration can then be transferred to the other detectors using the HEX source.

\subsection{Synchrotron calibration of a silicon photodiode}

The silicon photodiode used by NSTec for calibrations of camera efficiency that is described in Section 6 was manufactured by International Radiation Detectors (IRD) who claimed that its measurements are absolute (IRD Reference). This claim was substantiated by sending 
two photodiodes to the German synchrotron at the PTB. The photodiode efficiency was measured from $1 \mathrm{keV}$ to $60 \mathrm{keV}$. The measured efficiency was $100 \%$ from $1000 \mathrm{eV}$ up to the Si K edge at $1839 \mathrm{eV}$, then dropped about 3\% to $97 \%$ due to the Si escape photon. The efficiency rose back to $100 \%$ by $3.5 \mathrm{keV}$. The efficiency begins to fall near $5 \mathrm{keV}$ as the absorption of the Si falls below $100 \%$.

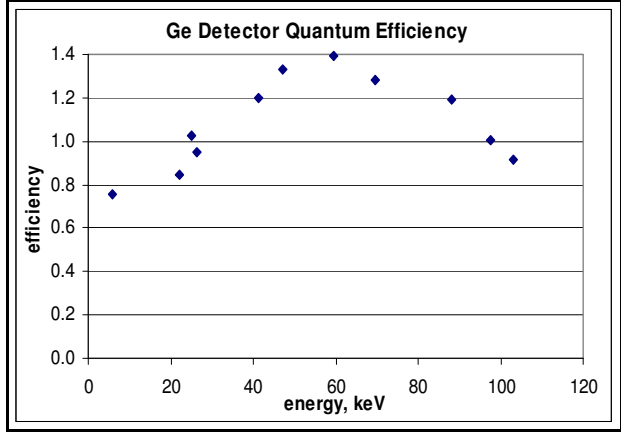

(a)

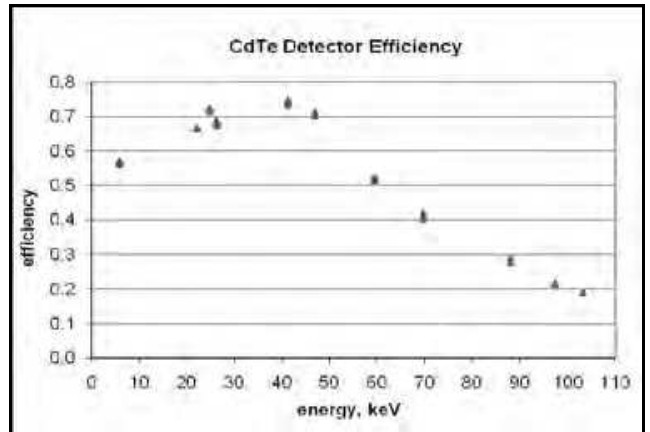

(b)

Fig. 14. Measurements of the quantum efficiency of the (a) Ge detector and (b) CdTe detector using the radionuclide sources.

NSTec has performed calibrations using the IRD Si photodiode, accepting the concept that it was an absolute photodiode and using the IRD measured thickness to correct for X-ray transmission at the higher energies. PTB calibration is an internationally accepted standard. The 2 calibrated photodiodes are then utilized as primary standards to cross calibrate other photodiodes which will be our working devices.

\subsection{Accreditation procedure for calibration laboratories at NSTec}

In order to provide substantiated traceability to international standards that would verify the quality of the NSTec calibration laboratories, we have been working with the National Voluntary Laboratory Accreditation Program (NVLAP). NVLAP is one of several organizations that can provide the certification that calibration procedures meet national and international standards. The NSTec laser timing laboratories have obtained this accreditation. The US National Institute for Standards and Technologies (NIST) does not have standards for X-ray intensity measurements; their concentration has been dosimetry. The calibration methods described previously are the starting point in the NSTec effort to achieve accreditation for the X-ray laboratories. Through the development of an accredited X-ray intensity calibration methodology, the diagnostics and system components for pulsed high energy physics experiments conducted at the national laboratories such as LLNL can now be calibrated traceable to national standards. Since the NSTec had already passed the NVLAP accreditation process on specific measurements in two laser laboratories, the groundwork for the X-ray laboratories was already done. The HEX laboratory would be the first one to be accredited. The required rigor of the quality assurance management system is already in place and approved. The required steps for accreditation are:

1. Determination of customer requirements

2. Development of calibration procedures and documentation of the procedures

3. Traceability 
4. Evaluation of measurement reproducibility

5. Determination of uncertainty

6. Generation of calibration certificates.

The main customer requirement is to have the capability to calibrate customer detectors and components to an absolute value with known uncertainties.

The previous sections have described the detector calibration procedure used to calibrate our X-ray source. The radionuclides used are traceable to NIST, and the vendor of these radionuclides participates in a measurement assurance program with NIST. NIST regularly sends standard radionuclide sources to the vendor to conduct "blind" measurements; the activity of the sources is unknown to the vendor. The vendor then measures the activity and submits the results back to NIST. NIST then checks the measurements to verify that they meet the required accuracy. The distance measurement traceability was described earlier.

As with all traceable measurements, repeatability and reproducibility determination are requirements for accreditation. The variation in repeated measurements quantifies random errors. Only when calibrations using a common procedure and common equipment produce the results that vary within an acceptable range, even with different operators, is the technical process is ready for NVLAP review.

NSTec is in the process of evaluating the overall uncertainty in its calibration of X-ray detectors with radionuclide sources. A list of the factors being considered is given in Table 9 . The procedures are being written at the time of this publication.

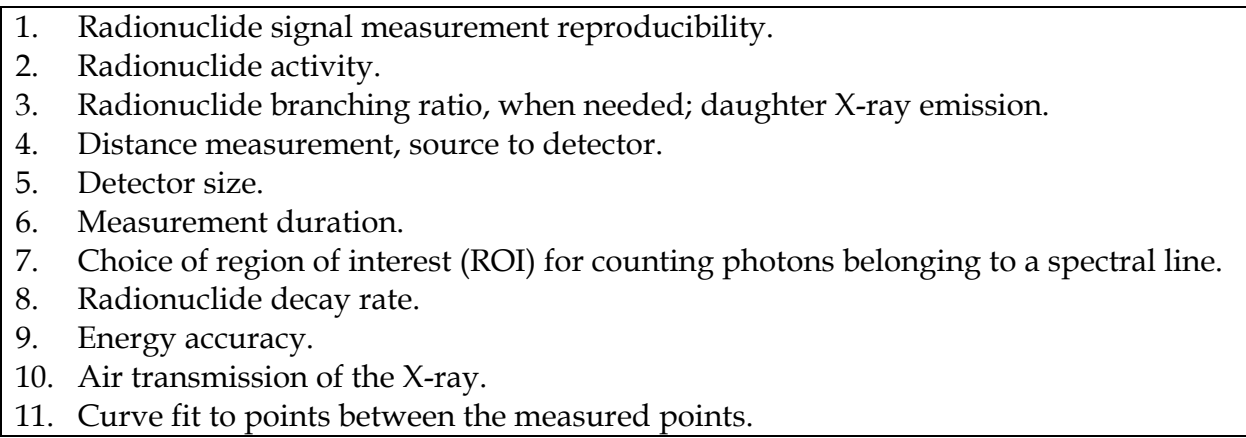

Table 9. Quantities in the Calibration Procedure Contributing to the Overall Uncertainty

\section{Calibrating X-ray cameras using the primary detectors}

\subsection{Measuring the quantum efficiency and its spatial variation (flat field) for an X-ray CCD camera using the Manson source}

The Static X-Ray Imager (SXI) is an X-ray camera used on the NIF target chamber to measure quantities such as laser beam pointing and the sizes of the laser entrance hole in ignition targets (Schneider, 2010). The SXI records a time integrated X-ray image. The sensor is a back thinned CCD chip with $2 \mathrm{kx} 2 \mathrm{k}$ pixels, $24 \mu \mathrm{m}$ square (approximately $50 \mathrm{~mm} \times 50$ $\mathrm{mm})$. The use of this camera at NIF requires the knowledge of camera sensitivity $\mathrm{S}(\mathrm{E})$ as a function of X-ray energy E.

The X-ray photon interacts with the Si sensor to produce hole-electron pairs that the CCD electronics process to produce the digital signal count $\mathrm{S}$. The number of electron-hole pairs produced by an X-ray photon that interacts with the Si sensor is a function of the photon 
energy and is slightly dependent upon the temperature (Janesick, 2000). The sensor is cooled to $253 \mathrm{~K}$ when operating.

A useful model relating the camera signal to fundamental quantities is found in Janesick (2000).

$$
\mathbf{S}(\mathrm{E})=\mathbf{P} \bullet \mathbf{A}_{\mathrm{pix}} \cdot \mathbf{T} \cdot \boldsymbol{\eta} \bullet \mathbf{Q E} \bullet \mathrm{K}^{-1} \text { counts } / \text { pixel }
$$

$\mathrm{P}=\quad$ Photon rate at the $\mathrm{CCD}$, photons $/ \mathrm{cm}^{2} / \mathrm{sec}$

$\mathrm{A}_{\text {pix }}=$ Pixel area

$\mathrm{T}=\quad$ Exposure time

$\mathrm{QE}=\quad$ Quantum efficiency, fraction of photons that interact with the pixel

$\eta=\quad$ Quantum yield, number of electron-hole pairs produced by the photon

$\mathrm{K}=\quad$ Camera gain, electron-hole pairs per count

For the Si CCD, the quantum yield is given by (Knoll, 2001):

$$
\eta=E / 3.66 \text { hole pairs/photon }
$$

The camera manufacturer's measurement of the gain constant was used for the calculations of quantum efficiency. The quantity being determined by the calibration is $\mathrm{S}(\mathrm{E})$, but the model given by Equation 10 is a valuable check of the calibration procedure, the calibration implementation, and is useful for troubleshooting camera problems. For the Si based cameras, the QE is related to the properties of $\mathrm{Si}$ as we shall see in the following results.

The photon intensity $\mathrm{P}^{\prime}$ was measured using a photodiode. We use a photodiode manufactured by IRD, model AXUV100. These detectors are designed with no doped dead region and zero surface recombination, so that they have near theoretical quantum efficiencies for the soft X-ray spectral region. One unit of this model was sent by the photodiode manufacturer for calibration to the synchrotron at the PTB. The results from this calibration showed agreement within $1 \%$ from $1000 \mathrm{eV}$ to $1839 \mathrm{eV}$, and the photodiode gave a $5 \%$ lower reading above this energy. The thickness of the Si photodiode is $54.5 \mu \mathrm{m}$. The current $\mathbf{i}$ from the photodiode is related to the photon intensity as given in Equation 12:

$$
\mathrm{i}=\mathrm{P}^{\prime} \cdot \eta_{\mathrm{PD}} \cdot 1.6 \times 10^{-19} \mathrm{amp}
$$

Here $\eta_{\mathrm{PD}}=\mathrm{E} / 3.62$ and $1.6 \times 10^{-19}$ is the charge on the elctron in coulombs. The area of the IRD detector is exactly $1 \mathrm{~cm}^{2}$. The photodiode and the CCD are not at the same distance from the anode, as can be seen in Fig. 6 . These distances were measured to an uncertainty of $2 \mathrm{~mm}$. The camera sensitivity $\xi$, defined as counts per photon:

$$
\begin{gathered}
\xi=S(E) /\left(P \bullet A_{\text {pix }} \bullet T\right) \\
\xi=S(E) /\left(\left(i /\left(\eta_{P D} \bullet 1.6 \times 10^{-19}\right)\right) \cdot\left(d_{\text {IRD }} / d_{C C D}\right)^{2} \bullet A_{\text {pix }} \bullet T\right)
\end{gathered}
$$

$\mathrm{P}=\quad$ Photon intensity at $\mathrm{CCD}$, photons $\bullet \mathrm{cm}^{-2} \bullet \mathrm{s}^{-1}$

$\mathrm{d}_{\mathrm{IRD}}=\quad$ Distance from the photodiode to the anode

$\mathrm{d}_{\mathrm{CCD}}=\quad$ Distance from the CCD to the anode

Note that $\mathbf{P}^{\prime}$, the photon intensity at the photodiode in Eq. 12 , is given by:

$$
\mathbf{P}^{\prime}=\mathbf{P} \bullet\left(\mathrm{d}_{\mathrm{IRD}} / \mathrm{d}_{\mathrm{CCD}}\right)^{2}
$$




\subsubsection{Methods for imaging}

The SXI's CCD camera was mounted on the diagnostic arm is shown Fig. 6. There was an extension between the camera and the Manson chamber of sufficient length that the X-ray beam uniformly illuminated the CCD. The camera calibration proceeded by the following steps:

1. Locate the bad pixels so that they can be masked out for image analysis;

2. Determine the linear range of the camera;

3. Measure the camera sensitivity;

4. Measure the uniformity of the CCD chip response over the area of the camera.

The cameras had a large number of bad rows and hot pixels. The bad rows were associated with the readout and identified using closed shutter images with a $3 \mathrm{~ms}$ exposure time. The hot pixels were identified by taking an image using the Ti anode and no filter, and using the same exposure time that was used for the experiments on the NIF target chamber experiments. A map was made that identified the bad rows and bad pixels.

The photon intensity was measured with the photodiode in arm \#1 as seen in Fig. 6. An exposure time was chosen to be as short as possible to give a reasonable signal. Photodiode readings were taken before and after acquiring each CCD image. During imaging, the X-ray beam intensity was monitored continuously for beam fluctuations using the photodiode in arm \#2. If there were beam intensity fluctuations observed during imaging, that image was discarded.

Flat field images are images where the CCD is uniformly illuminated in order to measure the uniformity of the camera response over its area. They were taken using the same anode voltage that was used for the camera efficiency measurements and maximum anode current. The exposure time was chosen to produce a signal that was $50 \%$ to $60 \%$ of saturation. Ten flat field images and ten background images were taken at each photon energy.

\subsubsection{Image analysis}

The camera images for the efficiency analysis had the background subtracted and the bad pixels replaced by the average of adjacent pixels. The mean pixel count was determined by randomly selecting 1000 regions 20x20 pixels in size, calculating the mean counts/pixel for each region and calculating the average of the means for each region. This is the signal $S$ for that image. Then, for the flat field images, average all images that have the same exposure time, average the background images, and subtract the average background from the average flat field image.

\subsubsection{Camera sensitivity}

The camera sensitivity for one of the SXI cameras is given in Fig. 15(a). The Quantum Efficiency (QE) calculated using Eq. 10 through 14 and camera gain $\mathrm{K}=7.62$ electrons per count is plotted as a function of photon energy in Fig. 15 (b). The data scatter as measured by the standard deviation was $1 \%$ or less at each point. The dip near $1800 \mathrm{eV}$ and the falloff after $2000 \mathrm{eV}$ are properties of $\mathrm{Si}$. Si that is $15 \mu \mathrm{m}$ thick transmits up to $35 \%$ as it approaches the $\mathrm{K}$ edge at $1839 \mathrm{eV}$. It begins transmitting again above $2500 \mathrm{eV}$ and is transmitting $80 \%$ at $8 \mathrm{keV}$. These QE results are similar to that obtained by Poletto (1999). There are two possible causes why the QE does not approach 1 when the photons are completely absorbed: (1) There may be absorption at the surface coating of the $\mathrm{Si}$; (2) the 
Quantum Yield may be less than the photon energy divided by $3.66 \mathrm{eV}$ per electron-hole pair. Analysis of a large number of single photon events could show the relative contribution of each effect.

\subsection{Flat field}

The flat field source is the $1 \mathrm{~mm}$ diameter spot on the anode. The anode is $1405 \mathrm{~mm}$ from the CCD. This arrangement would produce a flat field within $1 \%$ if there were nothing between the anode and the CCD. There is a light blocker that has an aluminum coating on a polyimide film (Al $1054 \AA \pm 50 \AA$; polyimide $1081 \AA \pm 100 \AA$ ). This item does not affect the flat field within the $1 \%$ cited above. The filter can cause a variation in the beam intensity across the CCD if there is sufficient variation in thickness, foreign material, or misalignment with the anode. A comparison of all the flat field images implies that the maximum variation is $\pm 1 \%$ peak-to-peak.

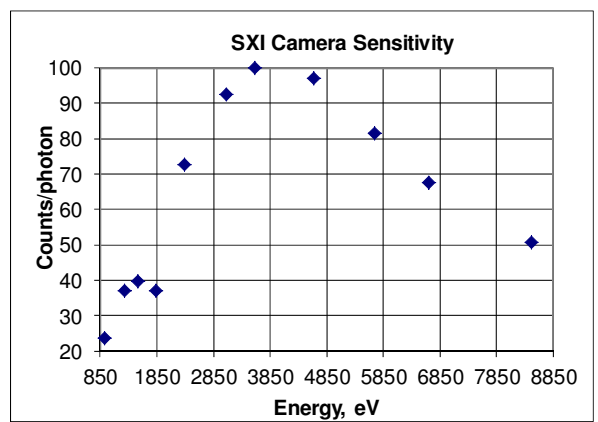

(a)

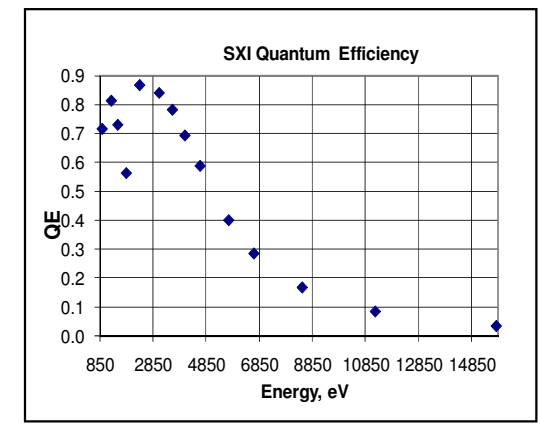

(b)

Fig. 15. The SXI (a) camera sensitivity and (b) quantum efficiency as measured by the camera count per pixel for each photon of a given energy. The measurements made at $\mathrm{X}$-ray energies below $8800 \mathrm{eV}$ were done on the Manson. The higher energy measurements were done on the HEX.

Fig. 16(a) shows the flat field image for one of the SXI cameras at the $\mathrm{Cu} 8470 \mathrm{eV}$ energy band. The image is set at high contrast so that the pixel signal variation shows clearly. A gross pattern is observed with the sensitivity at a maximum near the left center and decreasing slowly going away from the maximum. The image in Fig. 16 (b) is at Ti 4620 eV; it shows the same pattern but decreased magnitude. The pattern continues to decrease in magnitude until it is no longer visible at $3000 \mathrm{eV}$. Vertical lineouts averaged over a small horizontal width (see band in Fig. 16(b)) for three images at three different X-ray energies are shown in Fig. 17. The lineouts are normalized by dividing by the maximum counts in each image. The maximum sensitivity variation for each of the curves in Fig. 17 is $13 \%$ at $8470 \mathrm{eV}, 6 \%$ at $4620 \mathrm{eV}$ and $2 \%$ at $3580 \mathrm{eV}$.

A flat field image of the $\mathrm{Mg} 1275 \mathrm{eV}$ band is shown in Fig. 16(c) for comparison to the higher energy flat field images. There is no trace of the sensitivity variation pattern that is seen at higher energies. The $1275 \mathrm{eV}$ lineout in Fig. 17 shows that the maximum variation is less than $1 \%$, which is the measurement limit of our flat field procedure.

This sensitivity variation is a large scale effect; it includes groups of pixels and is probably related to the CCD manufacturing process. Any sensitivity variation of individual pixels is less than the photon noise associated with averaging 10 images. 
A different phenomenon was seen at low energies. Small irregular patches having diminished sensitivity were observed that are readily seen in Fig. 18(a). This image shows a portion of the CCD. The effect on sensitivity in these regions also shows an energy dependence. Fig. $8 \mathrm{~b}$ is a similar image taken at $3080 \mathrm{eV}$. The irregular patches have now become quite dim compared to what was observed at $1275 \mathrm{eV}$. At $4500 \mathrm{eV}$, these paths of low sensitivity have completely disappeared.

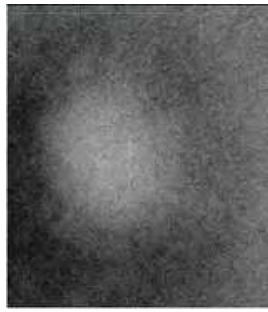

(a)

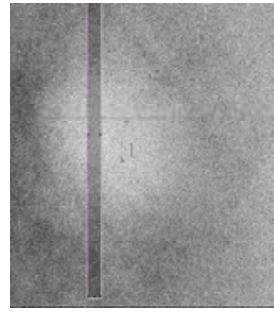

(b)

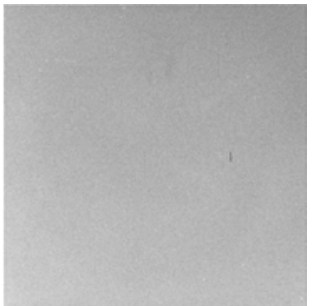

(c)

Fig. 16. Flat field image for the (a) $\mathrm{Cu}$ anode, $8470 \mathrm{eV}$ and (b) Ti anode, $4620 \mathrm{eV}$, showing the pixel sensitivity variation (Signal range: 5200 to 7200 counts/pixel) The vertical band was the area used to calculate the cross section that is shown in Fig. 17. The same region was used for the cross section at the other energies. (Signal range: 5200 to 7200 counts/pixel) Flat field image for the $\mathrm{Mg}$ anode, $1275 \mathrm{eV}$, showing the pattern observed at the higher energies shown in Fig. 16(a) and (b) has completely gone and the pixel sensitivity is flat.

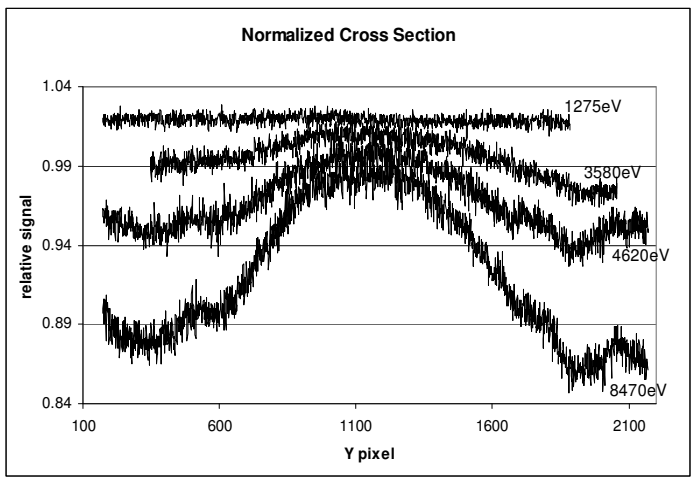

Fig. 17. Normalized vertical lineouts from flat field images at several X-ray energies. The lineouts were normalized to the maximum counts in each image. As the X-ray energy increases, the pixel sensitivity shows a greater vatiation.

There are several possible causes for these dark regions. Debris on the CCD surface could absorb X-rays and would be energy dependent, absorbing X-rays less as the energy increased. Damage to the CCD would likely cause an energy dependence that would increase the variance of the defective region from the surrounding pixels as the energy increased. Damage to the surface coating could produce this effect if the coating were thicker in that defective region. When we examined the CCD surface with a magnifying glass it did appear that the coating was deformed. It looked like a manufacturing defect. 
It is difficult to correct these images using the normal method of flat field inversion. This could be done if you limit the energy range of the X-ray source. But the characterization always provides the information necessary for the effective use of the $\mathrm{X}$-ray camera.

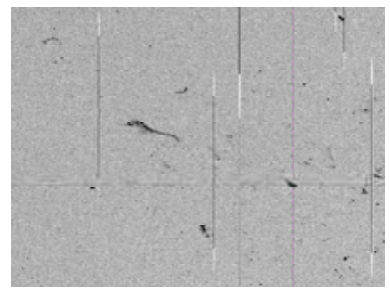

(b) $1275 \mathrm{eV}$

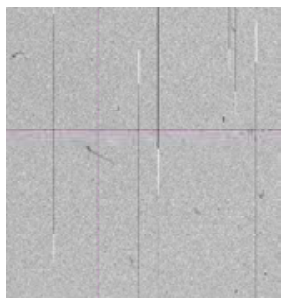

(a) $3080 \mathrm{eV}$

Fig. 18. These are the same sections of a flat field image taken at two different energies, (a) $1275 \mathrm{eV}$ and (b) $3080 \mathrm{eV}$. The sections cover about $1 / 4$ of the entire CCD. The dark regions are CCD surface defects causing diminished pixel sensitivity. For the $1275 \mathrm{eV}$ section shown in (a) the blemishes are much darker than in the $3080 \mathrm{eV}$ image shown in (b).

\subsection{Calibrating a front illuminated CCD camera from $705 \mathrm{eV}$ to $22 \mathrm{keV}$ using the Manson and HEX sources}

The SXI camera described above plays a critical role in the NIF operation, but this specific chip is no longer manufactured. There is another chip on the market with this large array, $2 \mathrm{kx} 2 \mathrm{k}, 24 \mu \mathrm{m}$ square, and we were requested to test the chip in a standard camera. The major concern regarding this chip was that it is front illuminated.

The QE measurements at X-ray energies below $10 \mathrm{keV}$ were done using the Manson source following the procedures given in 6.1. These measurements are shown in the graph of Fig. 15. Compare this to the results shown in Fig. 19 for the QE of the back illuminated camera. The maximum QE for the front illuminated camera is $Q E=0.34$ near $2300 \mathrm{eV}$. This is almost a factor of 3 lower than the QE measured for the back illuminated camera. The predominant difference begins to show below $1000 \mathrm{eV}$. At the $\mathrm{Cu} L$ lines, near $930 \mathrm{eV}$, the QE for the front illuminated camera is down by a factor of 10 from the front illuminated camera. At the Fe $\mathrm{L}$ lines near $705 \mathrm{eV}$, the QE is down by a factor of 100 .

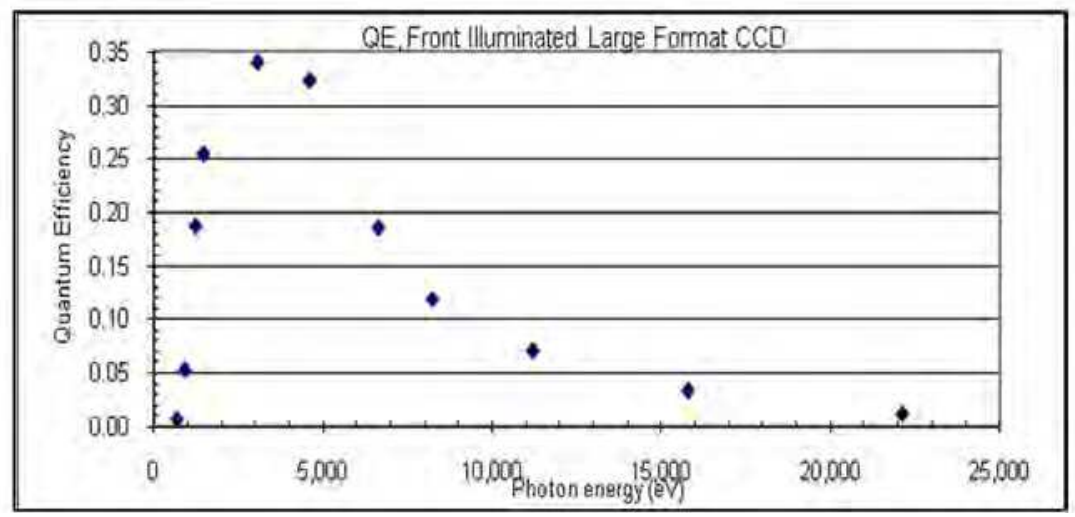

Fig. 19. The quantum efficiency measured for a front illuminated CCD sensor. 
The measurements at $10 \mathrm{keV}$ and lower energies were done on the Manson. The measurements at higher energies were made using the HEX. Compare this to the QE measurements shown in Fig. 15.

The Manson can only be used effectively up to the $\mathrm{Cu} \mathrm{K}$ lines. The QE measurements at higher energies have to be done on the HEX. The CCD cameras must be kept in a vacuum since they are cooled and the HEX has a vacuum chamber on a rail as is seen in Fig.11. The chamber is very similar to that shown on the Manson. It differs in having a Be window on the side facing the Hex source. The camera is mounted on the opposite side from the Be window. The HEX fluorescer source is near $10 \mathrm{~mm}$ diameter rather than the "point" source of the Manson. The X-ray beam is not flat across the entire CCD surface but is flat near the beam center. The camera is moved horizontally and vertically until the X-ray beam is centered on the CCD. The camera is then moved aside on the rail and the CdTe detector is placed at the same distance from the source as was the CCD. The beam center is then determined by moving the detector horizontally and vertically. These are the measurements used in Eq. 10 to determine the QE shown in Fig. 15 and Fig. 19 for the higher X-ray energies. The observation then is that the $\mathrm{QE}$ at these energies is the same for the front illuminated and the back illuminated cameras.

Measuring the sensitivity variation on the HEX requires that the X-ray intensity measurement be carefully measured over the entire area and an analytical representation be developed. This functionality is being developed now. We will use both the CdTe detector on a motorized $\mathrm{X}, \mathrm{Y}$ positioner and image plates to measure the $\mathrm{X}$-ray intensity distribution.

\subsection{Single photon measurements using the Manson source}

Images can be taken at sufficiently short exposure times so that most or all of the incidents recorded by the camera are caused by individual photons. These single photon images provide spectral information. This technique is used for astronomical measurements and laser plasma studies. The image shown in Fig. 20(a) was taken on the Manson source using a $\mathrm{Ti}$ anode and a Ti filter $100 \mu \mathrm{m}$ thick. This is the same condition that was used to generate the spectrum shown in Fig. 7 using an energy dispersive detector. The camera used was a silicon CCD type having 1300 pixel x 1340 pixel array and the pixel size was $13 \mu \mathrm{m}$ square. A background image using the same exposure time and no X-rays has been subtracted from the original $X$-ray image. The region shown in the figure is a 100 pixel square. There are approximately 95 single photon events in this 10000 pixel area, or about a 1\% fill. This is the fill rate typically used in single photon measurements. Note that a significant fraction of the single photon events produce counts in more than one pixel, that is, the production of electron/hole pairs produces by the photon occurs in more than one pixel.

The graph shown in Fig. 20(b) is a histogram of the entire pixel array for the single photon image of the Ti X-rays. This plot shows the number of times a pixel has a given count as a function of counts. The histogram exhibits two peaks and they are above 400 camera counts. The two peaks are the Ti Ka photons occurring at 415 camera counts and the Ti K $\beta$ photons occurring at 454 camera counts. These peaks represent single pixel events where the total number of electron/hole pairs produced by the photon is contained within that single pixel. As stated in the previous paragraph, there are many incidents in the image where the single photon produces counts in multiple pixels. These multi-pixel events produce the rising number of incidents in the graph going toward lower counts. There are no incidents at counts above the K-M band. Compare this spectrum to that shown in Fig. 7 where an energy 
dispersive Si detector was used. The spectral resolution is nearly the same for each detector. In general then, a camera is an energy dispersive detector when operated in the single photon mode.

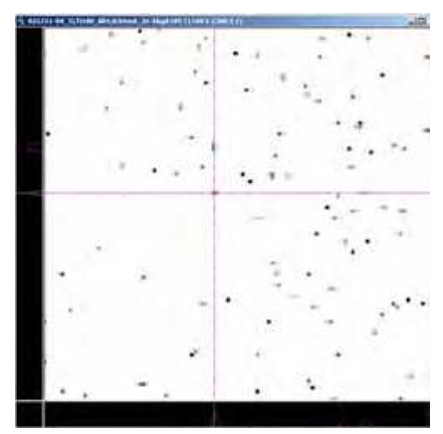

(a)

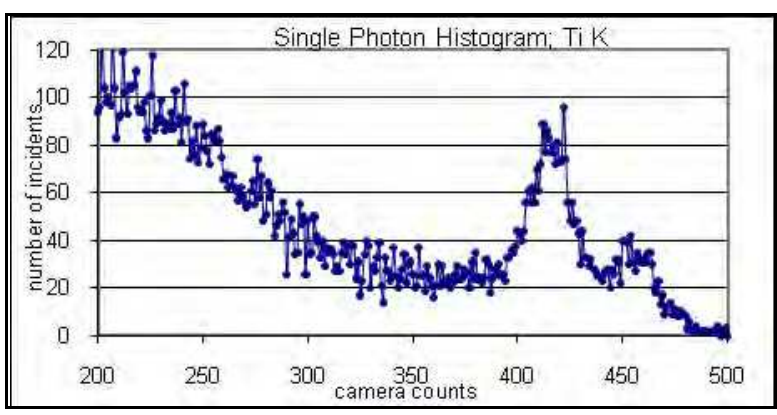

(b)

Fig. 20. (a) This image shows single photon incidents on a CCD camera zoomed in to show the individual pixels in a small region of the camera active area. (b) This graph is a CCD active area showing the Ti K-L and K-M spectral bands. Compare this to the spectral scan of the Ti emission using the energy dispersive detector shown in Fig. 3.

The above description also describes a method for calibrating the camera count to spectral energy. As described earlier for the camera efficiency calibration, images are taken with several anode/filter combinations. The camera count for the peak center is then plotted against the literature value for the spectral energy (more precisely, a weighted average of the unresolved spectral lines).

More sophisticated software than a simple histogram can be devised that would capture a large portion of the multi-pixel incidents that are single photon events. This would reduce the noise that is seen in the histogram peaks. The method requires identifying significant pixels by a thresholding technique, then adding the counts of adjacent pixels to the central pixel. This represents a new image that generates a new histogram. The spectral peaks will be better defined because the noise is reduced.

\subsection{Characterizing and calibrating an uncooled X-ray CID camera using the HEX source}

This section describes the characterization of a CID camera that was planned as the detector in a spectrometer system that was to be used on the LLNL NIF target chamber. The initial interest was to measure the emission from highly ionized Ge so the camera was characterized in the $10 \mathrm{keV}$ region using the HEX source (Carbone, 1998 and Marshall, 2001). The fluorescers chosen were $\mathrm{Cu}, \mathrm{Ge}$, and $\mathrm{Rb}$ giving weighted average for the $\mathrm{K}-\mathrm{L}$ and K-M transitions of $8.13 \mathrm{keV}, 10.01 \mathrm{keV}$, and $13.58 \mathrm{keV}$ respectively.

The major use for this CID sensor is for dental X-rays. It is relatively cheap and therefore expendable, a desirable property for the NIF application. The camera operates at room temperature normally, which gave a challenging problem to the characterization on HEX. Since the CID operates at room temperature, the dark current can saturate the camera for exposure times less than 10 seconds. This not a problem on NIF since the exposure time can be less than 1 second with sufficient $X$-rays to provide a bright spectral image. 
As indicated in the earlier description of CCD camera calibrations on the HEX, minutes of exposure time are needed to get a satisfactory signal. Preliminary experiments with the CID camera showed that we would be limited to three-second exposure times. It was determined that multiple exposures, on the order of 100 exposures, would be needed to obtain satisfactory photon statistics. The multiple exposures would also allow us to average the readout noise and get to the limit that photon statistics were dominant. A shutter control system was implemented for automatically taking the multiple images. We quickly found that drift in the dark current required us to take background images immediately after the $\mathrm{X}$-ray exposure. The system was designed so that an image was taken with the shutter open to the X-rays, then the next image was taken with the shutter closed. In this way a pair of images were produced, one image exposed to X-rays and the other as a background, that were close enough in time that there was no observable dark current drift. A black Kapton sheet, $50 \mu \mathrm{m}$ thick, was used to shield the camera from visible light. The same type shield is used for the camera on the NIF target chamber.

The X-ray beam was characterized geometrically using image plates to optimize collimator and distance choices. The intensity distribution was measured using the CdTe energy dispersive detector at multiple locations across the beam. Multiple images were taken with the CID, and then the detector was placed at the same location as the center of the CID had been located to verify that there was no drift in the X-ray source intensity. The multiple images were analyzed by subtracting each background from the previously taken X-ray image and summing the 100 resulting images. The final image then was effectively a 300 second exposure with the background removed. The measurements concentrated on the X-ray beam center for this initial effort. The CID camera efficiency, counts per pixel per photon, could then be calculated using the CdTe intensity measurements.

The results are shown in Fig. 21. The camera response was measured for two CID cameras at three spectral energies over the range of interest. The responses of the two cameras are the same within the experimental uncertainty. The expected response was modeled using the vendor's specification for camera gain and Si thickness and a typical surface coating. This is shown by the blue line in the figure. This did not fit the measurement data so a second model curve is shown using a thinner Si effective thickness.

The CID camera is now considered to be suitable for the spectrometer operation. The spectrometers will be incorporated as part of existing diagnostics at several locations on the NIF target chamber. All cameras will be calibrated using an extension of the procedure. It will extend to lower X-ray energies using the Manson source and measure the sensitivity variation of the CID over the full pixel array.

\section{Conclusion}

The chapter started with a presentation of basic X-ray physics needed to follow the description of X-ray detector calibration. The X-ray sources used at NSTec for calibrating detectors were described. The operation and characteristics of solid state semiconductor detectors was presented. Single sensor photodiodes, both current detectors and pulse counters, are used to measure the X-ray source beam intensities. The detectors are calibrated using either of 2 procedures: radioactive sources that are NIST traceable; a synchrotron beam that has an internationally accepted beam intensity accuracy. The chapter presented the methods used and the results obtained for calibrating several types of X-ray cameras. 


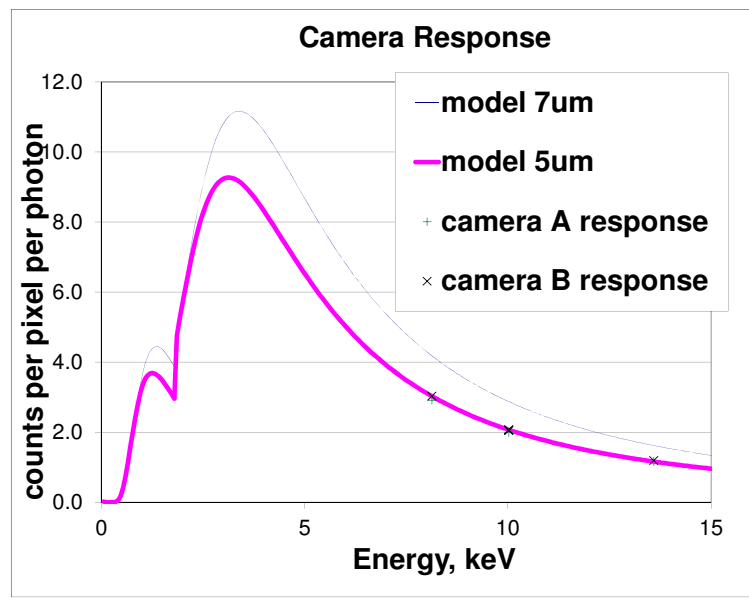

Fig. 21. The measurement results for the CID camera efficiency are shown as the crosses and the plus signs. The curves are model calculations for the CID camera response based on camera characteristics described in the text.

The accreditation procedure for recognition of the X-ray calibration labs as certified to international standards is in process. This requires the full analysis of all uncertainties associated with the detector calibration. The calibrated photodiode has yet to be completed for the synchrotron calibration. It will then be used to better fill the efficiency curves of the energy dispersive photodiodes. There are several agencies around the world that oversee and certify the accreditation. NSTec will be working with one of them to achieve certification. The NSTec X-ray labs will continually improve existing procedures and develop new methods for calibrating $X$-ray detection systems and components.

\section{Acknowledgment}

This manuscript has been authored by National Security Technologies, LLC, under Contract No. DE-AC52-06NA25946 with the U.S. Department of Energy. The United States Government and the publisher, by accepting the article for publication, acknowledges that the United States Government retains a non-exclusive, paid-up, irrevocable, world-wide license to publish or reproduce the published form of this manuscript, or allow others to do so, for United States Government purposes. This manuscript was done under the auspices of the U.S. Department of Energy by Lawrence Livermore National Laboratory under Contract DE-AC52-07NA27344. 
There were many persons from both NSTec and LLNL involved in developing the X-ray laboratory calibration methods. I particularly thank Susan Cyr for special effort in putting this manuscript together.

\section{References}

American Association of Physicists in Medicine (AAPM) (2006). Report No. 93, Acceptance Testing and Quality Control of Photostimulable Storage Phosphor Imaging Systems, available from http://www.aapm.org/pubs/reports/rpt_93.pdf

Carbone, J., Zulfiquar, A., Borman, C., Czebiniak, S., \& Ziegler, H. (1998). Large format CID x-ray image sensors, Proceedings of SPIE 3301, 90 doi:10.1117/12.304550, Solid State Sensor Arrays: Development and Applications II

Center for X-Ray Optics (CXRO) (n.d.). X-ray interactions with Matter, available from http://henke.lbl.gov/optical_constants/

ESTAR Program (n.d.). Available from http:// physics.nist.gov/PhysRefData/Star/Text/ESTAR.html

Gottwald, A., Kroth, U., Krumrey, M., Richter, M., Scholze, F., \& Ulm, G. (2006). The PTB high accuracy spectral responsivity scale in the VUV and x-ray range, Metrologia 43

Haugh, M. J. and Stewart, R. (2010). Measuring Curved Crystal Performance for a High Resolution Imaging X-ray Spectrometer, Hindawi Publishing

Haugh, M.J. \& Stewart, R. (2010). X-Ray Optics and Instrumentation, Article ID 583620

Herzberg, G. (1945). Atomic Spectra and Atomic Structure, Dover

International Radiation Detectors (IRD) (n.d.). Available from http:/ / www.ird-inc.com/axuvhighnrg.html

Janesick, J. (2000). Scientific Charge-Coupled Devices, SPIE Press, Bellingham, WA

Knoll, G. F. (2001). Radiation Detection and Measurement, $3^{\text {rd }}$ edition, John Wiley \& Sons

Maddox, B. et al (2011). High-energy backlighter spectrum measurements using calibrated image plates, RSI 82, 023111

Marshall, F. J., Ohki, T., McInnis, D., Ninkov, Z., Carbone, J. (2001). Imaging of laser-plasma x-ray emission with charge-injection devices, Rev. Sci. Instru. 72713

Poletto, L., Boscolo, A., \& Tondello, G. (1999), Characterization of a Charge-coupled Detector in the 1100-0.14 nm (1 eV to $9 \mathrm{keV})$ Spectral Range, Applied Optics, 38, 1 Jan 99

Physikalisch-Technische Bundensanstalt (PTB) (n.d.). available at http://www.ptb.de/index_en.html

Podgorsak, E. (2010). Radiation Physics for Medical Physicists $2^{\text {nd }}$ edition, Springer

Quaranta, C., Canali, G., Ottavani, G. , \& Zanio, K. (1969). Electron-hole Pair Ionization Energy in CdTe between $85 \mathrm{~K}$ and $350 \mathrm{~K}$, Lettere Al Nuovo Dimento, 4 , p. $908-910$

Schneider, M.B., Jones, O.S., Meezan, N.B. et al (2010). Images of lthe laser entrance hole from the static X-ray imager at NIF, Rev. Sci. Instru. 81 10E538.

Stepanov, S. (1997). X-ray Server, available from http://sergey.gmca.aps.anl.gov/ 
Stepanov, S. (2009). X0h Program, avalable from http://sergey.gmca.aps.anl.gov/x0h.html 


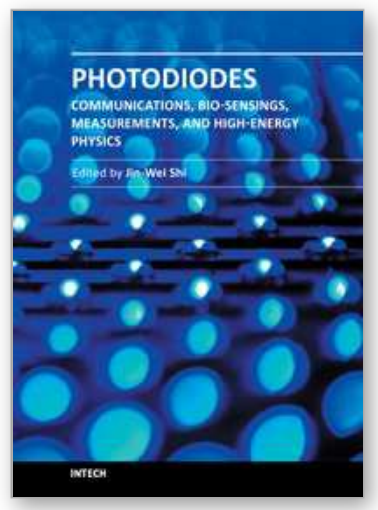

\author{
Photodiodes - Communications, Bio-Sensings, Measurements and \\ High-Energy Physics
}

Edited by Associate Professor Jin-Wei Shi

ISBN 978-953-307-277-7

Hard cover, 284 pages

Publisher InTech

Published online 06, September, 2011

Published in print edition September, 2011

This book describes different kinds of photodiodes for applications in high-speed data communication, biomedical sensing, high-speed measurement, UV-light detection, and high energy physics. The photodiodes discussed are composed of several different semiconductor materials, such as $\mathrm{InP}, \mathrm{SiC}$, and $\mathrm{Si}$, which cover an extremely wide optical wavelength regime ranging from infrared light to X-ray, making the suitable for diversified applications. Several interesting and unique topics were discussed including: the operation of highspeed photodiodes at low-temperature for super-conducting electronics, photodiodes for bio-medical imaging, single photon detection, photodiodes for the applications in nuclear physics, and for UV-light detection.

\title{
How to reference
}

In order to correctly reference this scholarly work, feel free to copy and paste the following:

Michael J. Haugh and Marilyn Schneider (2011). Quantitative Measurements of X-Ray Intensity, Photodiodes Communications, Bio-Sensings, Measurements and High-Energy Physics, Associate Professor Jin-Wei Shi (Ed.), ISBN: 978-953-307-277-7, InTech, Available from: http://www.intechopen.com/books/photodiodescommunications-bio-sensings-measurements-and-high-energy-physics/quantitative-measurements-of- $x$-rayintensity

\section{INTECH}

open science | open minds

\section{InTech Europe}

University Campus STeP Ri

Slavka Krautzeka 83/A

51000 Rijeka, Croatia

Phone: +385 (51) 770447

Fax: +385 (51) 686166

www.intechopen.com

\section{InTech China}

Unit 405, Office Block, Hotel Equatorial Shanghai

No.65, Yan An Road (West), Shanghai, 200040, China

中国上海市延安西路65号上海国际贵都大饭店办公楼405单元

Phone: +86-21-62489820

Fax: +86-21-62489821 
(C) 2011 The Author(s). Licensee IntechOpen. This chapter is distributed under the terms of the Creative Commons Attribution-NonCommercialShareAlike-3.0 License, which permits use, distribution and reproduction for non-commercial purposes, provided the original is properly cited and derivative works building on this content are distributed under the same license. 